\title{
Helicity amplitudes and electromagnetic decays of hyperon resonances
}

\author{
T. Van Cauteren ${ }^{1, a}$, J. Ryckebusch ${ }^{1}$, B. Metsch $^{2}$, and H.-R. Petry ${ }^{2}$ \\ 1 Ghent University, Department of Subatomic and Radiation Physics, Proeftuinstraat 86, B-9000 Gent, Belgium \\ 2 Helmholtz Institut für Strahlen- und Kernphysik, Nußallee 14-16, D-53115 Bonn, Germany
}

Received: 20 September 2005 / Revised version: 19 December 2005 /

Published online: 26 January 2006 - (c) Società Italiana di Fisica / Springer-Verlag 2006

Communicated by V. Vento

\begin{abstract}
We present results for the helicity amplitudes of the lowest-lying hyperon resonances $Y^{*}$, computed within the framework of the Bonn Constituent-Quark model, which is based on the Bethe-Salpeter approach. The seven parameters entering the model were fitted to the best-known baryon masses. Accordingly, the results for the helicity amplitudes are genuine predictions. Some hyperon resonances are seen to couple more strongly to a virtual photon with finite $Q^{2}$ than to a real photon. Other $Y^{*}$ 's, such as the $S_{01}(1670) \Lambda$-resonance or the $S_{11}(1620) \Sigma$-resonance, couple very strongly to real photons. We present a qualitative argument for predicting the behaviour of the helicity asymmetries of baryon resonances at high $Q^{2}$.
\end{abstract}

PACS. 11.10.St Bound and unstable states; Bethe-Salpeter equations - 12.39.Ki Relativistic quark model - 13.40.Gp Electromagnetic form factors - 14.20.Jn Hyperons

\section{Introduction}

The present work is part of an effort to develop a consistent description of kaon production processes of the type $p\left(\gamma, K^{+}\right) Y$ and $p\left(e, e^{\prime} K^{+}\right) Y \quad[1-3]$. Recent data for these processes are due to the CLAS Collaboration at Jefferson Laboratory [4], the LEPS Collaboration at SPring-8 [5], and the SAPHIR Collaboration at ELSA [6]. The abundant amount of new data calls for an adequate theoretical treatment. The availability of such a model appears indispensable for a proper interpretation of the experimental results, spanning an energy range from threshold up to $2.6 \mathrm{GeV}$.

One of the major sources of theoretical uncertainties when modeling $p\left(\gamma^{(*)}, K\right) Y$ reactions, is the strength of the electromagnetic (EM) couplings involved. This holds especially true for kaon electroproduction, where the EM coupling depends on $Q^{2}$, the squared four-momentum transfered by the virtual photon. The $Q^{2}$-dependence of the EM form factors is largely unknown for the "strange" baryons [3].

In a tree-level description of kaon electroproduction, the $\gamma^{*}-Y^{(*)}$ coupling comes into play in the $u$-channel (see fig. 1). The electromagnetic vertex is parameterized with the aid of elastic or transition form factors, which are input to isobar models. In ref. [7], we have presented our

\footnotetext{
a e-mail: Tim.VanCauteren@UGent.be
}

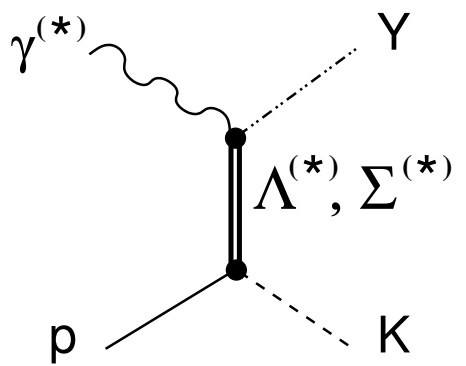

Fig. 1. The $u$-channel diagram with an exchanged hyperon or hyperon resonance in a tree-level isobar model for kaon production. The photon couples to the intermediate $Y^{(*)}\left(\Lambda^{(*)}\right.$, $\Sigma^{(*)}$ ), resulting in the outgoing (ground-state) hyperon $Y$.

results for the elastic form factors of ground-state hyperons and the form factors of the $\Sigma^{0}(1193) \rightarrow \Lambda(1116)$ electromagnetic transition, as computed in the framework of the Bonn Constituent-Quark (CQ) model [8-10]. In this work, we focus on the helicity amplitudes of hyperon resonances which decay electromagnetically to the groundstate $\Lambda$ and $\Sigma$ hyperons. These amplitudes are calculated in a parameter-free manner, and are compared with the (scarce) data to test the predictive power of the Bonn CQ model, as well as with previous calculations.

The Bonn CQ model also provides the EM form factors of other hadrons. Previous work has been reported for mesons and for nonstrange baryons. For the pseudoscalar- 
and vector-meson elastic and transition form factors [11], a fair description was reached both for data in the time-like and in the space-like region. For the pion, the outcome of the calculations was reasonable, considering the high values for the CQ masses in the model. For the nonstrange baryons [10], the results for the form factors and helicity amplitudes are reasonable to excellent.

There have been several attempts to predict the EM properties of hyperons and hyperon resonances. For the resonances, a number of theoretical studies for the photoand helicity amplitudes have been performed since the beginning of the eighties. These include studies of the EM decay widths and helicity amplitudes of the lowestlying hyperon resonances $\left(S_{01}(1405)\right.$ and $\left.D_{03}(1520)\right)$ to the octet $(\Lambda(1116)$ and $\Sigma(1193))$ and decuplet $\left(\Sigma^{*}(1385)\right)$ ground states within the context of a nonrelativistic CQ model [12] and a bag model [13]. A relativized CQ model was applied in ref. [14]. A treatment within the framework of the chiral bag model was presented in the early nineties by Umino and Myhrer in ref. [15]. More recent approaches adopt lattice QCD [16], heavy-baryon chiral perturbation theory [17], the bound-state soliton model [18, 19], the Skyrme model [20], the chiral constituent-quark model $[21,22]$, and the $1 / N_{c}$ expansion [23]. Most of these model calculations are restricted to the first and second hyperon resonance region (decuplet hyperons, $S_{01}(1405)$, and $\left.D_{03}(1520)\right)$. Note that the data on EM couplings indeed only cover those states [24]. Results for the other resonances are not constrained by data and should be interpreted as predictions or extrapolations.

Since only static EM properties of the lowest-lying hyperon resonances have been measured (the EM partial decay width), most of the aforementioned studies did not consider the $Q^{2}$-dependence of the helicity amplitudes. In addition, the validity of some models at intermediate and high momentum transfers is rather questionable. For $Q^{2} \simeq m^{2} \simeq m^{* 2}$, the hadron velocities in the lab frame is of the order $v^{2} / c^{2} \simeq 5 / 9$. This hints at sizeable boost effects and at the necessity of a Lorentz-covariant model. Also the validity of models based on chiral perturbation theory is restricted to momenta transfers smaller than a certain parameter, typically of the order of the mass of the nucleon.

In sect. 2 we will sketch how to compute baryon properties within the framework of the Bonn CQ model. This model is based on the Bethe-Salpeter approach, in which baryons are characterized by their Bethe-Salpeter amplitudes. The equation obeyed by this amplitude is presented in sect. 2.1. Solving this equation is far from trivial. Yet for instantaneous interactions (as used in the Bonn CQ model), the Bethe-Salpeter amplitude can be derived from the Salpeter amplitude. As discussed in sect. 2.2, this equation is more easily solvable. The calculation of the electromagnetic response of a hyperon resonance is the topic of sect. 3. Section 3.1 is devoted to the derivation of an expression for the current matrix elements within the Bonn model. The current matrix elements are then related to the helicity amplitudes of a hyperon resonance in sect. 3.2. In sect. 4 we will present the helicity amplitudes of the lowest-lying $\Lambda$-resonances for $0 \leq Q^{2} \leq 6 \mathrm{GeV}^{2}$, decaying electromagnetically to the $\Lambda(1116)$ (sect. 4.1) and to the $\Sigma(1193)$ (sect. 4.2 ) octet hyperons. The helicity amplitudes of the lowest-lying $\Sigma$-resonances (sect. 5) are presented in sect. 5.1 for the $\Sigma^{*}+\gamma^{(*)} \rightarrow \Lambda(1116)$ process and in sect. 5.2 for the $\Sigma^{*}+\gamma^{(*)} \rightarrow \Sigma(1193)$ process. In sect. 6 we will discuss the computed helicity asymmetries, especially for large $Q^{2}$. In sect. 7 we present our conclusions. The effective quark-quark interactions used in the Bonn model are given in appendix A.

\section{Baryons in the Bonn model}

The Bethe-Salpeter (BS) formalism outlined here, is based on the discussion of Le Yaouanc et al. [25]. It was described in great detail and applied to mesons and baryons in refs. [9-11]. In the Bonn CQ model, baryons are considered to be composed of three CQs. The three-CQ bound state is described by the BS amplitude. The basic idea of the formalism is to relate $n$-point Green's functions to the BS amplitudes of the particles under consideration. Through an ingenious application of the time-ordering operator, one isolates from the Green's function those terms which contain poles at those values of the kinematic variables where the particles are on shell. The residues of the Green's function at the poles are the products of the bound-state BS amplitudes and their adjoints. It then boils down to finding an equation for the Green's function which can be solved consistently to a certain order in the coupling constants of the interactions. In the course of this work, zeroth- and first-order approximations will be adopted.

\subsection{The Bethe-Salpeter equation}

In the Bonn CQ model, the basic quantity describing a baryon is the three-quark BS amplitude:

$$
\begin{aligned}
\chi_{\bar{P}, a_{1}, a_{2}, a_{3}}\left(x_{1}, x_{2}, x_{3}\right) \equiv \\
\left\langle 0\left|T\left(\Psi_{a_{1}}\left(x_{1}\right) \Psi_{a_{2}}\left(x_{2}\right) \Psi_{a_{3}}\left(x_{3}\right)\right)\right| \bar{P}\right\rangle,
\end{aligned}
$$

where $T$ is the time-ordering operator acting on the Heisenberg quark field operators $\Psi_{a_{i}}$, and $\bar{P}$ is the total four-momentum of the baryon with $\bar{P} \cdot \bar{P} \equiv \bar{P}_{\mu} \bar{P}^{\mu}=M^{2}$. The $a_{i}$ denote the quantum numbers in Dirac, flavour and colour space. For the sake of conciseness, these quantum numbers are frequently suppressed.

The BS amplitude is the solution of the BS equation for three interacting relativistic particles $[8,9,26]$. In momentum space, this equation reads:

$$
\chi_{\bar{P}}=-i G_{0 \bar{P}}^{(6)}\left(K_{\bar{P}}^{(3)}+\bar{K} \bar{P}_{\bar{P}}^{(2)}\right) \chi_{\bar{P}},
$$

where $\bar{P}$ is the on-shell momentum. In the equation above the arguments and indices have been suppressed. It is tacitly assumed that one integrates over arguments and sums 


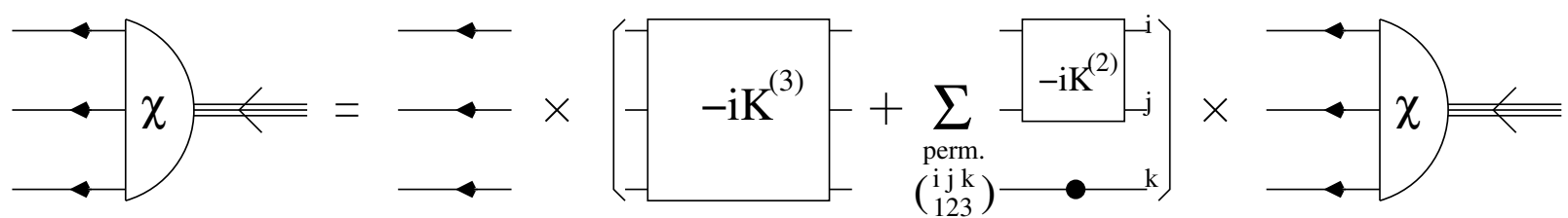

Fig. 2. The BS equation in a schematic form. Arrows represent quark propagators, the filled dot denotes an inverse propagator.

over indices that occur twice. The diagrammatical analogue of the BS equation for the amplitudes is shown in fig. 2. The normalization for the BS amplitudes is given by $[8,9]$

$$
-i \bar{\chi}_{\bar{P}}\left[P^{\mu} \frac{\partial}{\partial P^{\mu}}\left(G_{0 P}^{(6)^{-1}}+i K_{P}\right)\right]_{P=\bar{P}} \chi_{\bar{P}}=2 M^{2}
$$

In eq. (2) $G_{0 \bar{P}}^{(6)}$ is the direct product of the dressed propagators of the three quarks:

$$
\begin{aligned}
& G_{0 \bar{P}}^{(6)}\left(p_{\xi}, p_{\eta} ; p_{\xi}^{\prime}, p_{\eta}^{\prime}\right)=S_{F}^{1}\left(\frac{1}{3} \bar{P}+p_{\xi}+\frac{1}{2} p_{\eta}\right) \\
& \otimes S_{F}^{2}\left(\frac{1}{3} \bar{P}-p_{\xi}+\frac{1}{2} p_{\eta}\right) \otimes S_{F}^{3}\left(\frac{1}{3} \bar{P}-p_{\eta}\right) \\
& \times(2 \pi)^{4} \delta^{(4)}\left(p_{\xi}-p_{\xi}^{\prime}\right)(2 \pi)^{4} \delta^{(4)}\left(p_{\eta}-p_{\eta}^{\prime}\right),
\end{aligned}
$$

where the arguments are Jacobi momenta, as defined in ref. [7]. The propagators $S_{F}^{i}$ (with $i=1,2,3$ ) are approximated by those for constituent quarks:

$$
S_{F}^{i}\left(p_{i}\right)=\frac{i}{p_{i}-m_{i}+i \epsilon}
$$

where $m_{i}$ is the effective mass of the $i$-th constituent quark.

The quantity denoted by $K_{\bar{P}}^{(3)}$ in eq. (2) is the threeparticle irreducible interaction kernel for on-shell momenta $\bar{P}$. Further, $\bar{K} \bar{P}$ is a sum of two-particle irreducible interaction kernels, each multiplied by the inverse propagator of the spectator quark as can be seen in fig. 2 and in the expression

$$
\begin{aligned}
& \bar{K}_{\bar{P}}^{(2)}\left(p_{\xi}, p_{\eta} ; p_{\xi}^{\prime}, p_{\eta}^{\prime}\right)=K_{\left(\frac{2}{3} \bar{P}+p_{\eta}\right)}^{(2)}\left(p_{\xi}, p_{\xi}^{\prime}\right) \\
& \otimes\left[S_{F}^{3}\left(\frac{1}{3} \bar{P}-p_{\eta}\right)\right]^{-1}(2 \pi)^{4} \delta^{(4)}\left(p_{\eta}-p_{\eta}^{\prime}\right) \\
& + \text { cycl. perm. in quarks }(123) .
\end{aligned}
$$

In any CQ model, there exists some freedom with respect to the plausible types of interactions between the constituent quarks. We will use the instantaneous approximation. In the center-of-mass frame, the instantaneous approximation implies that the interaction kernels $K_{\bar{P}}^{(3)}$ and $K_{\left(p_{i}+p_{j}\right)}^{(2)}$ are independent of the energy components of the Jacobi momenta:

$$
\begin{aligned}
& \left.K_{\bar{P}}^{(3)}\left(p_{\xi}, p_{\eta} ; p_{\xi}^{\prime}, p_{\eta}^{\prime}\right)\right|_{\bar{P}=(M, \mathbf{0})} \equiv V^{(3)}\left(\mathbf{p}_{\xi}, \mathbf{p}_{\eta} ; \mathbf{p}_{\xi}^{\prime}, \mathbf{p}_{\eta}^{\prime}\right), \\
& \left.K_{\left(\frac{2}{3} \bar{P}+p_{\eta}\right)}^{(2)}\left(p_{\xi}, p_{\xi}^{\prime}\right)\right|_{\bar{P}=(M, \mathbf{0})} \equiv V^{(2)}\left(\mathbf{p}_{\xi}, \mathbf{p}_{\xi}^{\prime}\right) .
\end{aligned}
$$

We should mention here that whenever a quantity is to be evaluated in the rest frame of the baryon, we will indicate this by the index $M$, to make it clear that in this case $\bar{P}=(M, \mathbf{0})$.

The potentials used in our calculations are those of model $\mathcal{A}$ in ref. [27], since they provided the best results for the baryon spectrum. The three-particle interaction is given by a confinement potential $V_{\text {conf }}^{(3)}$ which rises linearly with interquark distances with an appropriate Dirac structure to avoid phenomenologically unwanted spin-orbit effects and as a residual interaction the 't Hooft InstantonInduced Interaction $V_{\text {III }}^{(2)}$ which acts between flavour antisymmetric quark pairs only [8]. The interaction potentials are discussed in more detail in appendix A.

The BS equation (2) and the normalization condition of eq. (3) for the BS amplitudes are Lorentz covariant. The transformation properties of the quantities involved are well known, so that if one can find a solution for, e.g., the BS amplitude in one Lorentz frame, it can be determined in an arbitrary frame. We will exploit the relativistic covariance of the model extensively by calculating quantities in the baryon's center-of-mass frame and boosting these to the desired frame in order to evaluate matrix elements.

\subsection{Reduction to the Salpeter equation}

The problem of solving eq. (2) is simplified by exploiting the instantaneous property of the interaction kernels because the integration over the energy components of the Jacobi momenta can be performed analytically. This gives rise to a new object $\Phi_{M}$, the Salpeter amplitude, which can be directly obtained from the full BS amplitude:

$$
\Phi_{M}\left(\mathbf{p}_{\xi}, \mathbf{p}_{\eta}\right)=\int \frac{\mathrm{d} p_{\xi}^{0}}{(2 \pi)} \frac{\mathrm{d} p_{\eta}^{0}}{(2 \pi)} \chi_{M}\left(\left(p_{\xi}^{0}, \mathbf{p}_{\xi}\right),\left(p_{\eta}^{0}, \mathbf{p}_{\eta}\right)\right) .
$$

The integration over the energy components is easily performed in situations where there are no genuine two-particle irreducible interactions in eq. (2), e.g. for the ground-state decuplet baryons which have symmetric spin wave functions. For other baryons, where the 


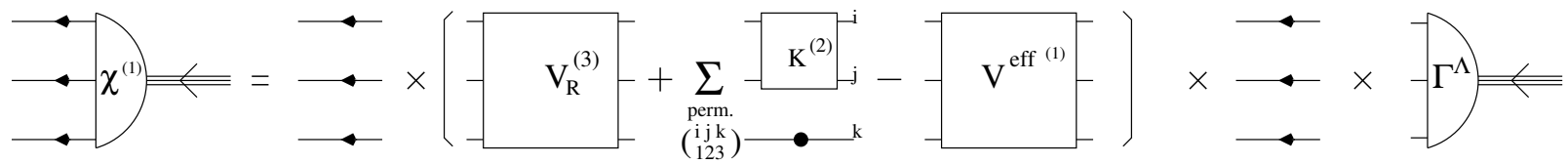

Fig. 3. The reconstruction of the BS amplitude from the vertex function according to eq. (15).

't Hooft Instanton-Induced Interaction $V_{\mathrm{III}}^{(2)}$ is nonvanishing, the inverse quark propagator in the two-particle kernel (eq. (6)) introduces an extra dependence on the energy components of the Jacobi momenta in the righthand side of eq. (2). This makes it impossible to do the integration analytically and a slightly different approach is needed, as is explained in ref. [9] and in the appendix of ref. [10]. There, it is pointed out that for reconstructing the Bethe-Salpeter amplitude defined in eq. (1), it suffices to compute the projection of the Salpeter amplitude of eq. (8) onto the purely positive-energy and negativeenergy states. This can be accomplished in the standard manner by introducing the energy projection operators:

$$
\Lambda_{i}^{ \pm}\left(\mathbf{p}_{i}\right)=\frac{\omega_{i}\left(\mathbf{p}_{i}\right) \mathbb{I} \pm H_{i}\left(\mathbf{p}_{i}\right)}{2 \omega_{i}\left(\mathbf{p}_{i}\right)},
$$

where $\omega_{i}\left(\mathbf{p}_{i}\right)=\sqrt{m_{i}^{2}+\left|\mathbf{p}_{i}\right|^{2}}$ denotes the energy and

$$
H_{i}\left(\mathbf{p}_{i}\right)=\gamma^{0}\left(\boldsymbol{\gamma} \cdot \mathbf{p}_{i}+m_{i}\right)
$$

is the free Dirac Hamiltonian for the $i$-th CQ. With the above definitions, one can project the Salpeter amplitude onto its purely positive- and purely negative-energy components,

$$
\begin{array}{r}
\Phi_{M}^{\Lambda}\left(\mathbf{p}_{\xi}, \mathbf{p}_{\eta}\right)=\left[\Lambda^{+++}\left(\mathbf{p}_{\xi}, \mathbf{p}_{\eta}\right)+\Lambda^{---}\left(\mathbf{p}_{\xi}, \mathbf{p}_{\eta}\right)\right] \\
\times \int \frac{\mathrm{d} p_{\xi}^{0}}{(2 \pi)} \frac{\mathrm{d} p_{\eta}^{0}}{(2 \pi)} \chi_{M}\left(\left(p_{\xi}^{0}, \mathbf{p}_{\xi}\right),\left(p_{\eta}^{0}, \mathbf{p}_{\eta}\right)\right)
\end{array}
$$

where $\Lambda^{+++}\left(\mathbf{p}_{\xi}, \mathbf{p}_{\eta}\right)=\Lambda_{1}^{+}\left(\mathbf{p}_{1}\right) \otimes \Lambda_{2}^{+}\left(\mathbf{p}_{2}\right) \otimes \Lambda_{3}^{+}\left(\mathbf{p}_{3}\right)$ and $\Lambda^{---}\left(\mathbf{p}_{\xi}, \mathbf{p}_{\eta}\right)=\Lambda_{1}^{-}\left(\mathbf{p}_{1}\right) \otimes \Lambda_{2}^{-}\left(\mathbf{p}_{2}\right) \otimes \Lambda_{3}^{-}\left(\mathbf{p}_{3}\right)$. After a tedious calculation [8], one obtains an equation for the projected Salpeter amplitude, which is given by

$$
\begin{aligned}
& \Phi_{M}^{\Lambda}\left(\mathbf{p}_{\xi}, \mathbf{p}_{\eta}\right)=\left[\frac{\Lambda^{+++}\left(\mathbf{p}_{\xi}, \mathbf{p}_{\eta}\right)}{M-\Omega\left(\mathbf{p}_{\xi}, \mathbf{p}_{\eta}\right)+i \varepsilon}\right. \\
& \left.+\frac{\Lambda^{---}\left(\mathbf{p}_{\xi}, \mathbf{p}_{\eta}\right)}{M+\Omega\left(\mathbf{p}_{\xi}, \mathbf{p}_{\eta}\right)-i \varepsilon}\right] \gamma^{0} \otimes \gamma^{0} \otimes \gamma^{0} \\
& \times \int \frac{\mathrm{d}^{3} p_{\xi}^{\prime}}{(2 \pi)^{3}} \frac{\mathrm{d}^{3} p_{\eta}^{\prime}}{(2 \pi)^{3}} V^{(3)}\left(\mathbf{p}_{\xi}, \mathbf{p}_{\eta} ; \mathbf{p}_{\xi}^{\prime}, \mathbf{p}_{\eta}^{\prime}\right) \Phi_{M}^{\Lambda}\left(\mathbf{p}_{\xi}^{\prime}, \mathbf{p}_{\eta}^{\prime}\right) \\
& +\left[\frac{\Lambda^{+++}\left(\mathbf{p}_{\xi}, \mathbf{p}_{\eta}\right)}{M-\Omega\left(\mathbf{p}_{\xi}, \mathbf{p}_{\eta}\right)+i \varepsilon}-\frac{\Lambda^{---}\left(\mathbf{p}_{\xi}, \mathbf{p}_{\eta}\right)}{M+\Omega\left(\mathbf{p}_{\xi}, \mathbf{p}_{\eta}\right)-i \varepsilon}\right] \\
& \times \int \frac{\mathrm{d}^{3} p_{\xi}^{\prime}}{(2 \pi)^{3}}\left[\left[\gamma^{0} \otimes \gamma^{0} V^{(2)}\left(\mathbf{p}_{\xi}, \mathbf{p}_{\xi}^{\prime}\right)\right] \otimes \mathbb{I}\right] \Phi_{M}^{\Lambda}\left(\mathbf{p}_{\xi}^{\prime}, \mathbf{p}_{\eta}\right)
\end{aligned}
$$

where $\Omega\left(\mathbf{p}_{\xi}, \mathbf{p}_{\eta}\right)$ is the sum of the energies of the three CQs in the center-of-mass frame,

$$
\Omega=\sum_{i=1}^{3} \omega_{i}=\sum_{i=1}^{3} \sqrt{\left|\mathbf{p}_{i}\right|^{2}+m_{i}^{2}} .
$$

In principle, one would need the full Salpeter amplitude to reconstruct the BS amplitude, but it turns out that the terms with the smallest denominators are exactly those with projector structures $\Lambda^{+++}\left(\mathbf{p}_{\xi}, \mathbf{p}_{\eta}\right)$ and $\Lambda^{---}\left(\mathbf{p}_{\xi}, \mathbf{p}_{\eta}\right)$. The denominators of the terms with other projector structures are large enough, so that these terms may safely be neglected [8].

Once the Salpeter equation (12) has been solved, the vertex function $\Gamma_{M}^{\Lambda}$ can be constructed:

$$
\begin{gathered}
\Gamma_{M}^{\Lambda}\left(\mathbf{p}_{\xi}, \mathbf{p}_{\eta}\right)=-i \int \frac{\mathrm{d}^{3} p_{\xi}^{\prime}}{(2 \pi)^{3}} \frac{\mathrm{d}^{3} p_{\eta}^{\prime}}{(2 \pi)^{3}}\left[V_{\Lambda}^{(3)}\left(\mathbf{p}_{\xi}, \mathbf{p}_{\eta} ; \mathbf{p}_{\xi}^{\prime}, \mathbf{p}_{\eta}^{\prime}\right)\right. \\
\left.+V_{M}^{\mathrm{eff}^{(1)}}\left(\mathbf{p}_{\xi}, \mathbf{p}_{\eta} ; \mathbf{p}_{\xi}^{\prime}, \mathbf{p}_{\eta}^{\prime}\right)\right] \Phi_{M}^{\Lambda,(1)}\left(\mathbf{p}_{\xi}^{\prime}, \mathbf{p}_{\eta}^{\prime}\right) .
\end{gathered}
$$

At first order, this vertex function describes how the three CQs couple to form a baryon. It can be related to the BS amplitude through

$$
\chi_{\bar{P}} \approx \chi_{\bar{P}}^{(1)}=\left[G_{0 \bar{P}}\left(V_{R}^{(3)}+\bar{K}_{\bar{P}}^{(2)}-V_{\bar{P}}^{\mathrm{eff}^{(1)}}\right) G_{0 \bar{P}}\right] \Gamma_{\bar{P}}^{\Lambda},
$$

of which a diagram is shown in fig. 3 .

In eqs. (14) and (15), we have defined

$$
\begin{aligned}
V_{\Lambda}^{(3)} & =\left(\gamma^{0} \otimes \gamma^{0} \otimes \gamma^{0}\right)\left(\Lambda^{+++}+\Lambda^{---}\right) \\
& \times\left(\gamma^{0} \otimes \gamma^{0} \otimes \gamma^{0}\right) V^{(3)}\left(\Lambda^{+++}+\Lambda^{---}\right),
\end{aligned}
$$

which is that part of the three-body potential which involves purely positive-energy and negative-energy components of the amplitudes only. Further, $V_{R}^{(3)}=V^{(3)}-V_{\Lambda}^{(3)}$ is the remaining part which involves the mixed-energy components only. $V_{\bar{P}}^{\text {eff }^{(1)}}$ is a first-order approximation of an effective potential with three-body structure which parameterizes the two-body interaction $[9,10]$. Further, $\bar{K} \bar{P}_{\bar{P}}^{(2)}$ is defined in eqs. (6) and (7b).

\section{Electromagnetic response}

In the Bonn CQ model, it is possible to calculate the matrix elements of any operator which can be written in terms of quark field creation and annihilation operators. We will focus on the Current Matrix Elements (CMEs) with one incoming and one outgoing baryon. The current operator used in this work describes EM transitions. The incoming and outgoing states are bound states of three constituent quarks, which are described by the BS amplitudes discussed in sect. 2 . In sect. 3.1 we will derive how 


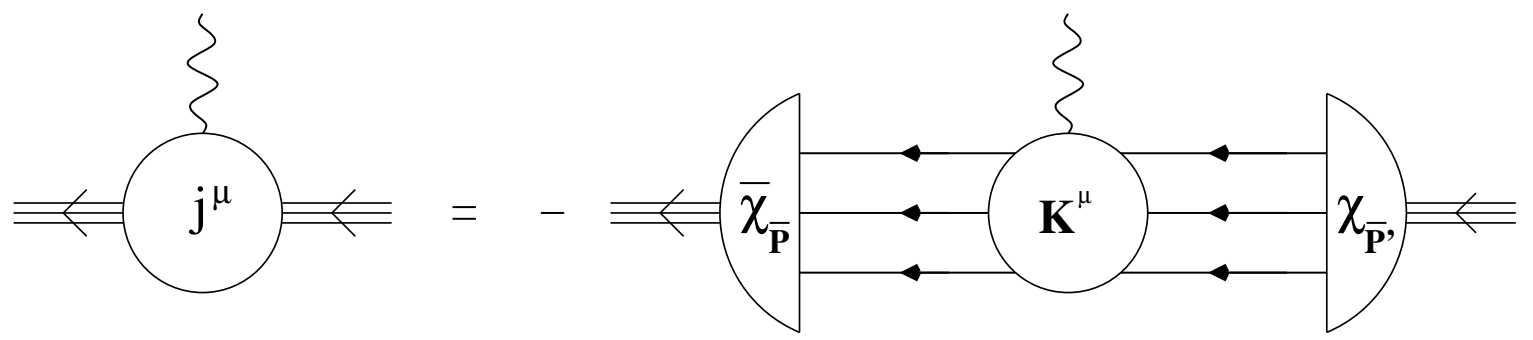

Fig. 4. Diagrammatic representation of eq. (18) for the CME.

the CMEs are related to the BS amplitudes. In a next step, we will derive the CMEs in terms of the lowest-order approximations to the BS amplitudes and the simplest expression for the photon coupling to a three-quark bound state. In sect. 3.2, the computed current matrix elements will be linked to the helicity amplitudes.

\subsection{Current matrix elements}

After determining the BS amplitudes and the corresponding vertex functions according to eqs. (14) and (15), the CMEs can be computed through the following definition of the current operator $j^{\mu}(x)$ :

$$
\left\langle\bar{P}\left|j^{\mu}(x)\right| \bar{P}^{\prime}\right\rangle=\left\langle\bar{P}\left|\bar{\Psi}(x) \hat{q} \gamma^{\mu} \Psi(x)\right| \bar{P}^{\prime}\right\rangle,
$$

where $\Psi$ and $\hat{q}$ are the CQ field in the Heisenberg picture and the charge operator, respectively. The current operator $j^{\mu}(x)$ corresponds to the photon coupling to a point-like CQ. At this point, we have refrained from using CQ form factors in order not to introduce any additional parameters. This might affect our results at high $Q^{2}$. The above matrix element can be expressed in terms of the objects defined in sects. 2.1 and 2.2 [10,28]:

$$
\begin{aligned}
& \left\langle\bar{P}\left|j^{\mu}(0)\right| \bar{P}^{\prime}\right\rangle=-\int \frac{\mathrm{d}^{4} p_{\xi}}{(2 \pi)^{4}} \frac{\mathrm{d}^{4} p_{\eta}}{(2 \pi)^{4}} \frac{\mathrm{d}^{4} p_{\xi}^{\prime}}{(2 \pi)^{4}} \frac{\mathrm{d}^{4} p_{\eta}^{\prime}}{(2 \pi)^{4}} \\
& \times \bar{\chi}_{\bar{P}}\left(p_{\xi}, p_{\eta}\right) K_{P ; q ; P^{\prime}}^{\mu}\left(p_{\xi}, p_{\eta} ; p_{\xi}^{\prime}, p_{\eta}^{\prime}\right) \chi_{\bar{P}^{\prime}}\left(p_{\xi}^{\prime}, p_{\eta}^{\prime}\right) .
\end{aligned}
$$

This equation can be most easily interpreted with the aid of the diagrams shown in fig. 4 .

Up to this point, we have not introduced any approximation concerning the order of the interactions. In acquiring the BS amplitudes within the framework of sect. 2, however, we restricted ourselves to the lowest order for the three-particle and two-particle irreducible kernels in order to make the equations analytically tractable and numerically computable. For a consistent calculation, a lowestorder approximation for the kernel $K^{\mu}$ appears necessary. The kernel $K^{\mu}$ in eq. (18) should thus be expressed in terms of one-quark propagators and interaction kernels up to lowest order. Using Wick's theorem, one can subsequently write all connected terms without any interactions contributing to the seven-point Green's function. We find 18 terms, which can be subdivided into three groups, which are interconnected by a permutation operator on the CQs. However, the BS amplitudes are antisymmetric by construction when interchanging two CQs, so the antisymmetric contributions in the kernel $K^{\mu}$ will be projected out. Therefore, only three terms will have to be taken into account, each of which describes the photon coupling to one of the CQs. The expression for $K^{\mu}$ up to zeroth order in configuration space reads [10]

$$
\begin{aligned}
& K^{\mu(0)}\left(x_{1}^{\prime \prime}, x_{2}^{\prime \prime}, x_{3}^{\prime \prime} ; x ; x_{1}^{\prime \prime \prime}, x_{2}^{\prime \prime \prime}, x_{3}^{\prime \prime \prime}\right)= \\
& S_{F}^{{ }^{-1}}\left(x_{1}^{\prime \prime}-x_{1}^{\prime \prime \prime}\right) \otimes S_{F}^{2}{ }^{-1}\left(x_{2}^{\prime \prime}-x_{2}^{\prime \prime \prime}\right) \\
& \otimes\left[\delta^{(4)}\left(x_{3}^{\prime \prime}-x\right) \hat{q} \gamma^{\mu} \delta^{(4)}\left(x-x_{3}^{\prime \prime \prime}\right)\right] \\
& + \text { cycl. perm. in quarks (123). }
\end{aligned}
$$

From this, the expression for the kernel in momentum space can be easily obtained.

In evaluating eq. (18), the kernel $K_{P ; q ; P^{\prime}}^{\mu}$ is considered up to lowest order, as in eq. (19). Exploiting the cyclic permutation symmetry of the latter, the CME is obtained easily

$$
\begin{aligned}
& \left\langle\bar{P}\left|j^{\mu}(0)\right| \bar{P}^{\prime}\right\rangle \simeq(-3) \int \frac{\mathrm{d}^{4} p_{\xi}}{(2 \pi)^{4}} \frac{\mathrm{d}^{4} p_{\eta}}{(2 \pi)^{4}} \bar{\chi}_{\bar{P}}^{(1)}\left(p_{\xi}, p_{\eta}\right) \\
& \times\left[S_{F}^{1-1}\left(\frac{P}{3}+p_{\xi}+\frac{p_{\eta}}{2}\right) \otimes S_{F}^{2}{ }^{-1}\left(\frac{P}{3}-p_{\xi}+\frac{p_{\eta}}{2}\right)\right. \\
& \left.\otimes \hat{q} \gamma^{\mu}\right] \chi_{\bar{P}^{\prime}}^{(1)}\left(p_{\xi}, p_{\eta}+\frac{2}{3} q\right),
\end{aligned}
$$

where $q$ is the (incoming) photon four-momentum. In the above equation, we are using the first-order approximation to the BS amplitudes from eq. (15). Instead of explicitly calculating the BS amplitudes with eq. (15) and inserting them into eq. (20), it is more convenient to express the CMEs in terms of the vertex functions. We insert the vertex functions $\Gamma_{\bar{P}}^{\Lambda}$ from eqs. (14) and (15) into our approximate formula for the CME and retain the lowest-order terms. Eventually, we arrive at $[10,28]$

$$
\begin{aligned}
& \left\langle\bar{P}\left|j^{\mu}(0)\right| M\right\rangle \simeq(-3) \int \frac{\mathrm{d}^{4} p_{\xi}}{(2 \pi)^{4}} \frac{\mathrm{d}^{4} p_{\eta}}{(2 \pi)^{4}} \bar{\Gamma} \frac{\Lambda}{P}\left(p_{\xi}, p_{\eta}\right) \\
& \times S_{F}^{1}\left(\frac{M}{3}+p_{\xi}+\frac{p_{\eta}}{2}\right) \otimes S_{F}^{2}\left(\frac{M}{3}-p_{\xi}+\frac{p_{\eta}}{2}\right) \\
& \otimes\left[S_{F}^{3}\left(\frac{M}{3}-p_{\eta}+q\right) \hat{q} \gamma^{\mu} S_{F}^{3}\left(\frac{M}{3}-p_{\eta}\right)\right] \\
& \times \Gamma_{M}^{\Lambda}\left(p_{\xi}, p_{\eta}+\frac{2}{3} q\right) .
\end{aligned}
$$




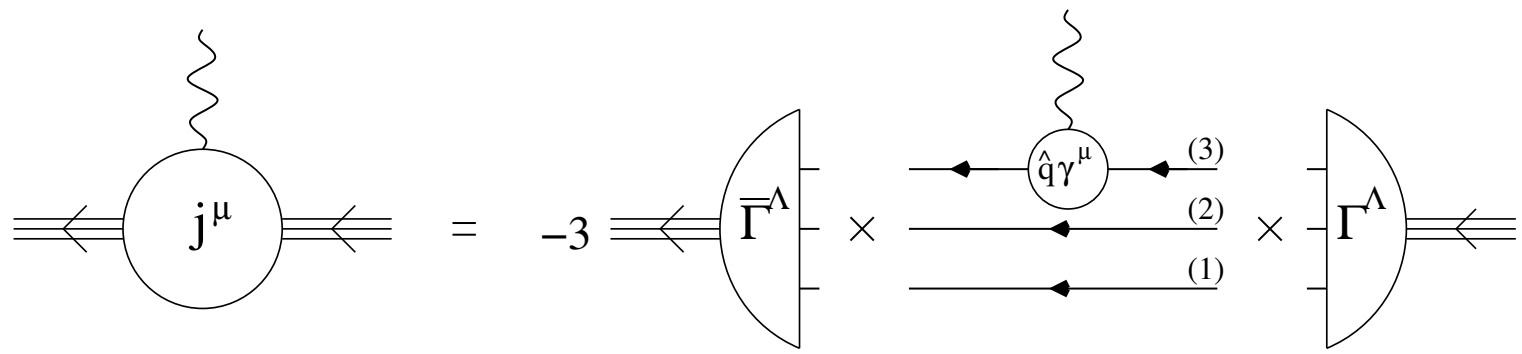

Fig. 5. Feynman diagram showing the coupling of the photon to the third CQ as in eq. (21). The other two CQs are spectators.

Here, $\bar{\Gamma} \bar{P}$ is the adjoint vertex function, calculated in the c.o.m. frame according to

$$
\bar{\Gamma}_{M}^{\Lambda}=-\left(\Gamma_{M}^{\Lambda}\right)^{\dagger} \gamma^{0} \otimes \gamma^{0} \otimes \gamma^{0}
$$

Under a Lorentz boost, this vertex function transforms as [28]

$$
\Gamma_{\bar{P}}\left(p_{\xi}, p_{\eta}\right)=S_{\Lambda}^{1} \otimes S_{\Lambda}^{2} \otimes S_{\Lambda}^{3} \Gamma_{\Lambda^{-1} \bar{P}}\left(\Lambda^{-1}\left(p_{\xi}\right), \Lambda^{-1}\left(p_{\eta}\right)\right),
$$

with $\Lambda$ the boost matrix and $S_{\Lambda}^{i}$ the corresponding boost operator acting on the $i$-th quark (not to be confused with the propagator of the $i$-th quark $S_{F}^{i}$ ). Equation (21) is a consistent lowest-order approximation of the CME. We refer the reader to refs. [10] and [28] for more details, and to fig. 5 for a schematic representation of eq. (21). After an appropriate treatment of the pole terms in the integration over the energy variables, in the remaining integral over $\mathbf{p}_{\xi}$ and $\mathbf{p}_{\eta}$, the azimuthal dependence can be reduced to $\left(\phi_{\xi}-\phi_{\eta}\right)$, leaving one with five-dimensional integrals, which are computed numerically.

\subsection{Helicity amplitudes}

The literature on EM decays of nonstrange-baryon resonances within the framework of a quark model is extensive [10,29]. For resonances, the concept of EM form factors as coefficients to EM-vertex structures is involved, especially for spin $J \geq 3 / 2$ resonances (see, e.g., ref. [30] for $J=3 / 2$ resonances). In general, the EM properties are parameterized in terms of Helicity Amplitudes (HAs). These quantities can be directly written in terms of the CMEs of the constituent-quark model.

Depending on the conventions made with respect to normalization factors, other definitions for the HAs emerge. Using the conventions of ref. [10], one gets for the EM transitions between excited $\left(B^{*}\right)$ and groundstate $(B)$ baryons,

$$
\begin{aligned}
& A_{1 / 2}\left(B^{*} \rightarrow B\right)= \\
& \quad \mathcal{D}\left\langle B, \bar{P}, \frac{1}{2}\left|j^{1}(0)+i j^{2}(0)\right| B^{*}, \bar{P}^{*},-\frac{1}{2}\right\rangle, \\
& A_{3 / 2}\left(B^{*} \rightarrow B\right)= \\
& \mathcal{D}\left\langle B, \bar{P},-\frac{1}{2}\left|j^{1}(0)+i j^{2}(0)\right| B^{*}, \bar{P}^{*},-\frac{3}{2}\right\rangle,
\end{aligned}
$$

$$
C_{1 / 2}\left(B^{*} \rightarrow B\right)=\mathcal{D}\left\langle B, \bar{P}, \frac{1}{2}\left|j^{0}(0)\right| B^{*}, \bar{P}^{*}, \frac{1}{2}\right\rangle,
$$

with $\mathcal{D}=\sqrt{\frac{\pi \alpha}{2 m\left(m^{* 2}-m^{2}\right)}}$. There are only two independent CMEs for $B^{*}\left(J^{*}=1 / 2\right) \rightarrow B(J=1 / 2)$ transitions, and three for $B^{*}\left(J^{*} \geq 3 / 2\right) \rightarrow B(J=1 / 2)$ transitions. With the above normalizations, the width corresponding to the EM decay of an excited state $B^{*}$ with mass $m^{*}$ to a ground-state baryon $B$ with mass $m$ and spin $J=1 / 2$, is given by

$$
\Gamma_{\gamma}=\frac{|\mathbf{q}|^{2}}{4 \pi^{2} \alpha} \frac{2 m}{\left(2 J^{*}+1\right) m^{*}}\left[\left|A_{1 / 2}\right|^{2}+\left|A_{3 / 2}\right|^{2}\right] .
$$

Here, $|\mathbf{q}|=\frac{m^{* 2}-m^{2}}{2 m^{*}}$ is the three-momentum of the photon in the rest frame of the initial baryon resonance, and $\alpha=\frac{e^{2}}{4 \pi} \simeq \frac{1}{137}$ is the EM fine-structure constant. The adopted definition for the EM decay width differs from the one given by the Particle Data Group (PDG) [24] by a factor of $e^{2}=4 \pi \alpha$. The PDG tables present the experimentally known EM decay widths and photo-amplitudes $A_{i}\left(Q^{2} \rightarrow 0\right)$. We compute the full $Q^{2}$-dependence of the EM properties of hyperon resonances in terms of HAs in sects. 4 and 5. However, we should remark that in the present calculation only the relative signs between the $A_{1 / 2}, A_{3 / 2}$ and $C_{1 / 2}$ of a certain $B^{*} \rightarrow B$ decay are fixed. For $\Sigma^{*} \rightarrow \Sigma$ decays, the relative HAs for the different isospin channels are also determined.

\section{Results for the $\Lambda$-resonances}

In this section, results are presented for the helicity amplitudes (HAs) of $\Lambda^{*}$-resonances decaying to the $\Lambda$ or $\Sigma$ ground-state hyperons. The HAs are defined in eqs. (24). We will organize our results according to the quantum numbers of the resonances and the ground-state hyperon to which they decay. Most of the computed low-lying states can be identified with experimentally known resonances by comparing the calculated with the experimental mass spectrum [31]. This is illustrated in fig. 6 for the $\Lambda^{*}$ spectrum. Only for the higher-lying $F_{05}(2110)$ and $D_{03}(2325)$, no direct correspondence with a single computed state can be made.

We will use the nomenclature adopted by the Particle Data Group (PDG) [24] to identify a state (e.g. $D_{03}(1520)$ for the lowest-lying $\Lambda$-resonance with $\left.J^{\pi}=3 / 2^{-}\right)$. In 


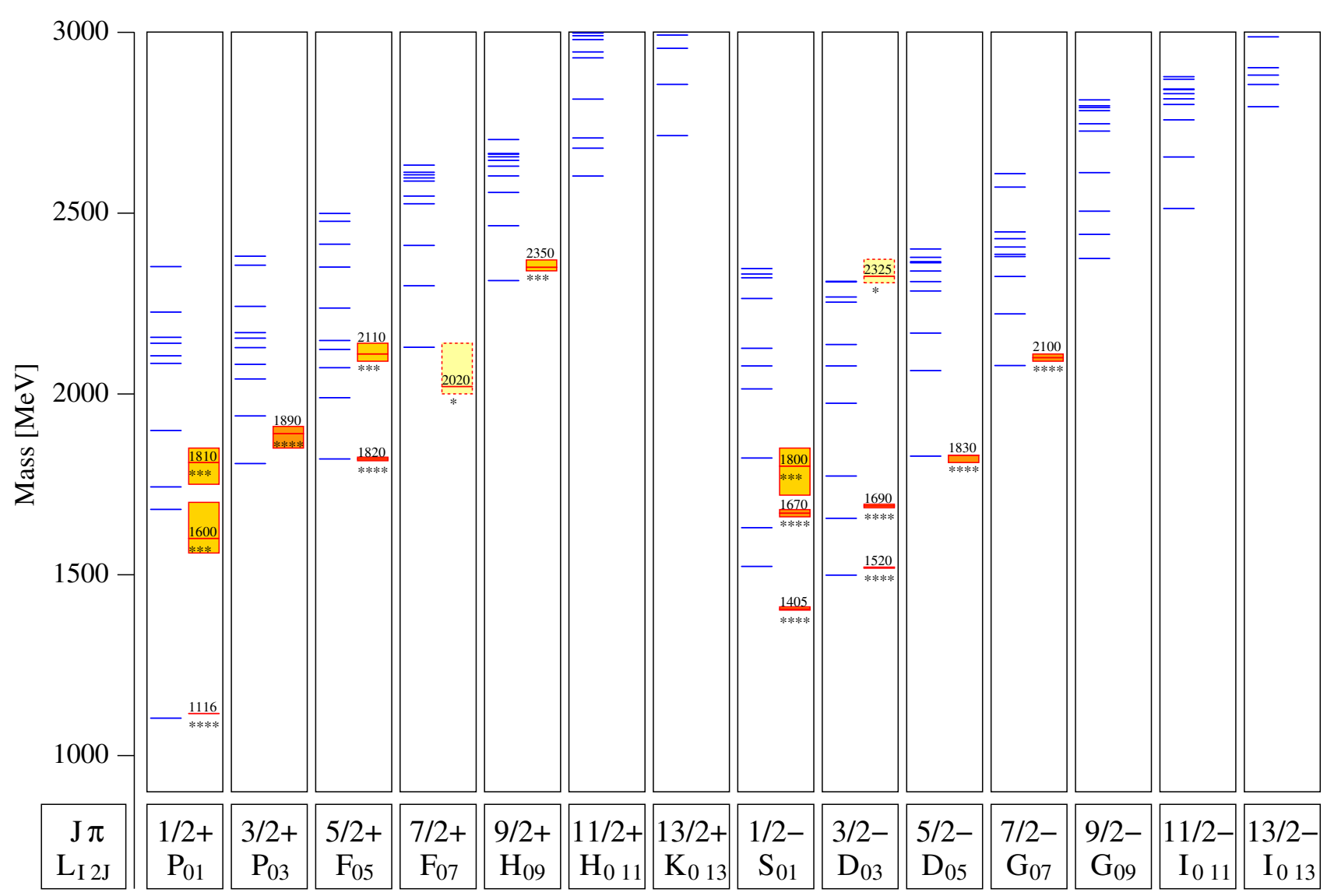

Fig. 6. The left part of each column displays the calculated $\Lambda^{*}$ spectrum [31] for positive- and negative-parity states with spins up to $J=13 / 2$ and masses up to $3000 \mathrm{MeV}$. The predictions are compared with the spectrum from ref. [24] (right part of each column). The stars indicate the confidence level for the existence of each state. The uncertainty on a mass is indicated by the shaded area.

those situations where there is no clear identification possible, the excited state with given quantum numbers will be labeled with a number. The lowest-lying resonance gets number "1", the second resonance "2", etc. Occasionally, the ground state will be identified with a " 0 ". (Note that what we call a ground state is a member of the baryon octet.)

To illustrate the notation conventions, consider the $\Lambda^{*}$ spectrum in fig. 6 . The ground state is denoted by $P_{01}^{(0)} \equiv P_{01}(1116)$. The first computed resonance, the $P_{01}^{(1)}$, can be identified with the experimentally observed $P_{01}(1600)$-resonance. For the $J^{\pi}=3 / 2^{-}$resonances, the two lowest computed states, the $D_{03}^{(1)}$ and the $D_{03}^{(2)}$, can be recognized as the measured $D_{03}(1520)$ and $D_{03}(1690)$ resonances, respectively. The third computed resonance is as yet unobserved experimentally, and will thus be called the $D_{03}^{(3)}$. Note that we use the PDG conventions for denoting the strange baryons: $L_{I, 2 J}$ with the isospin $I$, spin $J$, and $L=S, P, D, \ldots$, the orbital angular momentum of the partial wave in which the resonance could be observed in $\bar{K} \Lambda$ scattering.
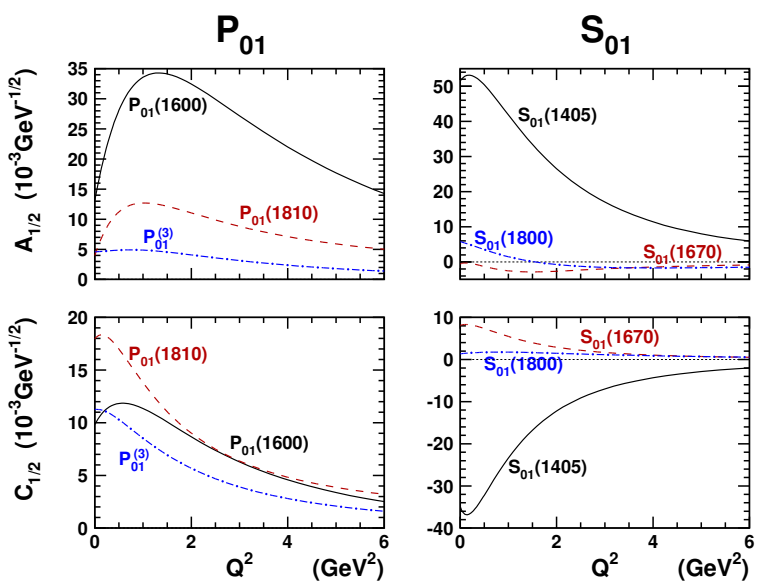

Fig. 7. The $Q^{2}$-dependence for the $\Lambda^{*}+\gamma^{*} \rightarrow \Lambda$ decays for spin $J=1 / 2$ resonances: left (right) panels show the results for the positive (negative)-parity $\Lambda^{*}$-resonances.

\section{$4.1 \Lambda^{*}+\gamma^{(*)} \rightarrow \Lambda$ transitions}

Our results for $\operatorname{spin} J=1 / 2$, isospin $I=0$ resonances are summarized in fig. 7. Already for the lowest-lying 
Table 1. Calculated masses, photo-amplitudes and EM decay widths for the $\Lambda^{*}+\gamma \rightarrow \Lambda(1116)$ transition. Values between brackets denote the experimental decay width of the $\Lambda$-resonance as given by ref. [24]. Masses and decay widths are given in units of $\mathrm{MeV}$, photo-amplitudes are given in units of $10^{-3} \mathrm{GeV}^{-1 / 2}$.

\begin{tabular}{|c|c|c|c|c|}
\hline Resonance & $M_{\text {calc }}$ & $A_{1 / 2}$ & $A_{3 / 2}$ & $\Gamma_{\text {calc }}$ \\
\hline \hline$P_{01}(1600)$ & 1752 & 13.0 & - & 0.104 \\
$P_{01}(1810)$ & 1805 & 3.97 & - & 0.0105 \\
$P_{01}^{(3)}$ & 1928 & 4.59 & - & 0.0174 \\
$S_{01}(1405)$ & 1550 & 51.5 & - & 0.912 \\
& & & & $(0.027 \pm 0.008)$ \\
$S_{01}(1670)$ & 1664 & -0.574 & - & $0.159 \times 10^{-3}$ \\
$S_{01}(1800)$ & 1879 & 5.76 & - & 0.0252 \\
\hline$P_{03}(1890)$ & 1834 & 18.3 & -5.58 & 0.129 \\
$P_{03}^{(2)}$ & 1970 & -17.7 & 2.90 & 0.142 \\
$P_{03}^{(3)}$ & 2068 & 3.33 & 12.9 & 0.0893 \\
$P_{03}^{(4)}$ & 2116 & 6.81 & 2.92 & 0.0293 \\
$D_{03}(1520)$ & 1511 & 5.50 & -41.2 & 0.258 \\
& & & & $\left(0.133_{-0.031}^{+0.034}\right)$ \\
$D_{03}(1690)$ & 1678 & 13.8 & 11.6 & 0.0815 \\
$D_{03}^{(3)}$ & 1805 & -6.31 & -18.8 & 0.130 \\
\hline$F_{05}(1820)$ & 1837 & 12.8 & -7.82 & 0.0531 \\
$F_{05}^{(2)}$ & 2012 & -7.74 & 3.41 & 0.0223 \\
$F_{05}^{(3)}$ & 2104 & 2.98 & 11.6 & 0.0503 \\
$D_{05}(1830)$ & 1843 & 11.3 & 16.0 & 0.0916 \\
$D_{05}^{(2)}$ & 2114 & 20.6 & -7.78 & 0.172 \\
$D_{05}^{(3)}$ & 2219 & 4.22 & -1.53 & 0.00805 \\
\hline
\end{tabular}

$Y^{*}$-resonances, one observes interesting features in the computed HAs. In the left panel of fig. 7, the HAs of the three lowest $J^{\pi}=\frac{1}{2}^{+} \Lambda$-resonances are displayed. The first-excited state with the same quantum numbers as the ground-state baryon is the analogue of the Roper resonance in the nucleon spectrum. In the $\Lambda$ spectrum, this state is observed experimentally with $m \approx 1600 \mathrm{MeV}$. For the computed state which can be identified with the $P_{01}(1600)$-resonance, the $A_{1 / 2}$ amplitude reaches its maximum at $Q^{2} \approx 1.5 \mathrm{GeV}^{2}$. Accordingly, the Roper-like resonance in the $\Lambda$ spectrum may not show up in photoproduction experiments, but only in electroproduction reactions at intermediate $Q^{2}$-values. Indeed, a space-like photon couples to the intermediate $Y^{*}$-resonance with a strength proportional to its HA at that specific $Q^{2}$. Signals of the $P_{01}(1600)$-resonance in electromagnetically induced kaon production are predicted to become particularly important at $Q^{2} \approx 1.5 \mathrm{GeV}^{2}$. Another interesting feature is that the $P_{01}(1810)$ has a relatively large $C_{1 / 2}$. The $C_{1 / 2}$ contributes to the longitudinal part of the kaon electroproduction strength. Optimum conditions to detect signals of the $P_{01}(1810)$ are thus created when looking at the longitudinal part of the $p\left(e, e^{\prime} K^{+}\right) \Lambda$ cross-sections at small $Q^{2}$.

The most striking observation for the $S_{01}$-resonances (right panels of fig. 7), is the dominance of the lowest excitation $S_{01}(1405)$. We denote this state with the exper-
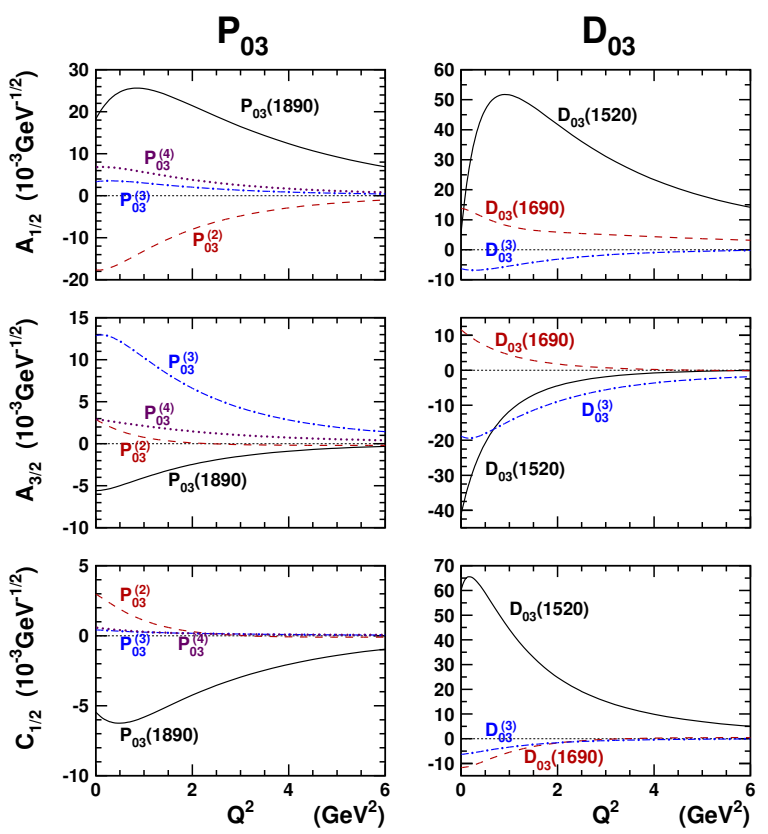

Fig. 8. The $Q^{2}$-dependence for the $\Lambda^{*}+\gamma^{*} \rightarrow \Lambda$ decays for spin $J=3 / 2$ resonances: left (right) panels show the results for the positive (negative)-parity $\Lambda^{*}$-resonances.

imental mass of the first excitation with quantum numbers $(J=1 / 2, S=-1, T=0)$ and negative parity, but from table 1 and fig. 6 , it is clear that its mass is not well reproduced. Also the calculated EM decay width of this state is too large by a factor about 50 . For the photon amplitude this implies a factor of 7 , which is a huge deviation considering the quality of our calculations for the magnetic moments of the ground-state hyperons [7]. We conclude that the $S_{01}(1405)$ is not well described in our CQ model. Possible explanations of this discrepancy is the inadequacy of the effective interactions used, strong rescattering effects with e.g. the $\bar{K} N$-channel, different degrees of freedom (a three-quark structure is possibly inadequate), a double-pole structure, etc. $[32,33]$. For the higher-lying $S_{01}$-resonances, our calculations predict very small electromagnetic couplings to the $\Lambda(1116)$.

The HAs for the lowest-lying spin $J=3 / 2 \Lambda^{*}$ 's are shown in fig. 8. In the left panels, we consider $P_{03}$ hyperons with positive parity. For the $A_{1 / 2}$, the $P_{03}(1890)$ reaches its maximum at $Q^{2} \simeq 1.0 \mathrm{GeV}^{2}$, after which it slowly falls to zero. The $P_{03}^{(2)}$ with a calculated mass of $1970 \mathrm{MeV}$ (cf. table 1 ), has a reasonably large $A_{1 / 2}$, but falls off rather quickly compared to the first resonance. The other HAs, the $A_{3 / 2}$ and the $C_{1 / 2}$, are rather small for the $P_{03}$ states. Only the $P_{03}^{(3)}$ with an expected mass of $2068 \mathrm{MeV}$ gives a modest signal in $A_{3 / 2}$.

The results for the $D_{03}$ helicity amplitudes are summarized in the right panels of fig. 8. Again, one notices a peak in the $Q^{2}$-dependence of the first resonance at $Q^{2} \simeq 0.8 \mathrm{GeV}^{2}$ for the $A_{1 / 2}$, and at $Q^{2} \simeq 0.2 \mathrm{GeV}^{2}$ for the $C_{1 / 2}$. Both HAs fall off slowly for large $Q^{2}$-values. 
Systematically, we find that for almost all $I$ and $J$, the first resonance $L_{I, 2 J}^{(1)}$ reaches a maximum in $A_{1 / 2}$ and $C_{1 / 2}$ at moderate values of $Q^{2}$. For $J \geq 3 / 2$, the $A_{3 / 2}$ 's reach their maximum at $Q^{2}=0 \mathrm{GeV}^{2}$, and show a gradual falloff with growing $Q^{2}$. Furthermore, the strongest coupling is reached at smaller values of $Q^{2}$ for negative-parity resonances than for positive-parity resonances.

It is not uncommon that CQ models predict helicity amplitudes or transition form factors which reach their maximum at moderate $Q^{2}[10,34,35]$. Since it is not the case for elastic form factors [7], one might deduce that its origin lies in the nonzero orbital momentum of the BS amplitudes for excited states. The overlaps of the latter with the BS amplitudes of ground states may then be maximal at finite $Q^{2}$. However, this is a delicate issue, which depends largely on the operator, on the BS amplitudes of incoming and outgoing baryons and on the boost involved in the scattering process.

The $D_{03}(1520)$ will couple quite strongly to virtual photons with $Q^{2} \approx 0.5 \mathrm{GeV}^{2}$. In a partial-wave analysis of $p\left(e, e^{\prime} K^{+}\right) \Lambda$ data, the first $D_{03}$-resonance is likely to overwhelm the effect of the higher-lying resonances with identical quantum numbers, which have only moderate HAs. In table 1 , one notices that for the $D_{03}(1520)$, the EM decay width is known up to a factor of roughly two. The computed value is about $50 \%$ larger than the upper limit of the experimental width. However, the EM decay width could be influenced by strong mixing effects with the $\bar{K} N$-channel (threshold around $1433 \mathrm{MeV}$ ), which are not included in the model.

In the isobar model developed for $p\left(\gamma^{(*)}, K\right) Y$ processes in refs. $[1-3,36]$, resonances up to $J \leq 3 / 2$ are included. Therefore, it is instructive to see whether there is evidence from CQ calculations to justify this approximation. The HAs for the $J=5 / 2$ hyperon resonances are shown in fig. 9. In the left panels, the HAs of the three lowest-lying states with quantum numbers $J^{\pi}=\frac{5}{2}^{+}$ are displayed. Again, one observes a pronounced maximum in the $A_{1 / 2}$ and $C_{1 / 2}$ for the $F_{05}(1820)$. If we consider the masses in table 1 , it is easily seen that the computed mass of the $F_{05}^{(2)}$ is too small for it to be identified with the experimentally observed $F_{05}(2110)$. As a matter of fact, from fig. 6 , it is clear that the third, fourth and fifth resonance have a (computed) mass approaching the experimentally determined value. In ref. [31], it is argued that the second resonance is actually a missing hyperon resonance and that the experimentally determined state around $2110 \mathrm{MeV}$ should be associated with one of the higher-lying $F_{05}$-resonances of a CQ model calculation. The smallness of the helicity amplitudes displayed in fig. 9 suggests that in photo- and electroinduced $\Lambda$ production processes, it is unlikely that the $F_{05}^{(2)}$ and $F_{05}^{(3)}$ $\Lambda^{*}$-resonances will result in strong background signals.

The right panels of fig. 9 contain the predictions for the three lowest-lying $D_{05}$-resonances. The first resonance can be associated with the $D_{05}(1830)$ state from ref. [24]. Like for the $S_{01}$ and $D_{03}$ resonances, the $A_{1 / 2}$ and $C_{1 / 2}$ reach their maximum at low, but finite $Q^{2}$-values. In con-
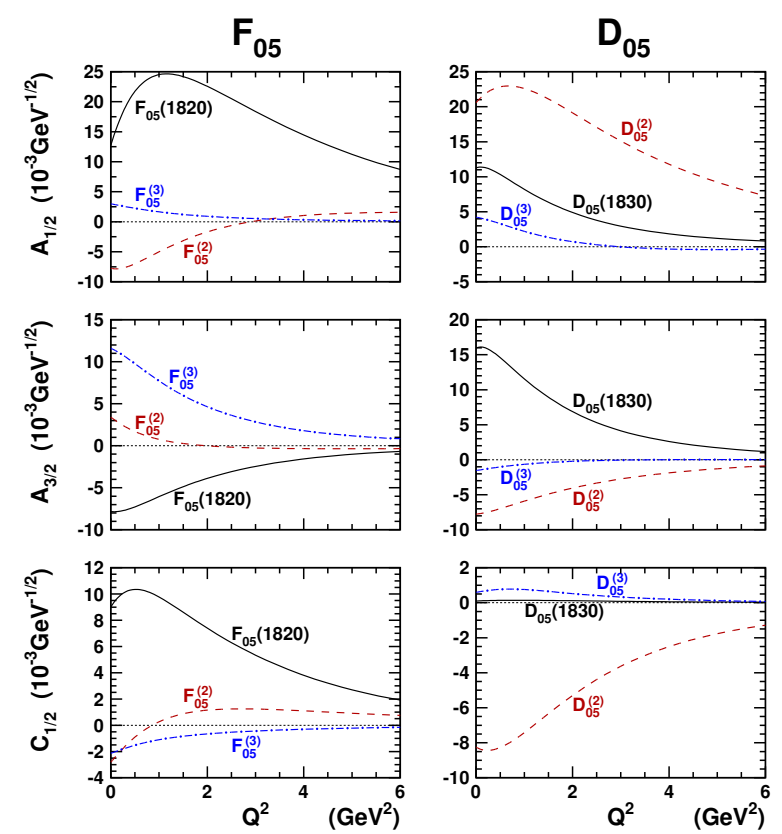

Fig. 9. The $Q^{2}$-dependence for the $\Lambda^{*}+\gamma^{*} \rightarrow \Lambda$ decays for spin $J=5 / 2$ resonances: left (right) panels show the results for the positive (negative)-parity $\Lambda^{*}$-resonances.

trast to the $J=1 / 2$ and $J=3 / 2 \Lambda^{*}$-resonances, the second $D_{05}$-resonance has larger HAs than the first resonance. The PDG tables do not mention evidence for this $D_{05}^{(2)}$ state [24]. On the basis of their computed HAs, the $F_{05}(1820)$, the $D_{05}(1830)$ and the $D_{05}^{(2)}$ resonances can be expected to contribute sizably to the $p\left(\gamma^{(*)}, K\right) \Lambda$ reaction dynamics. Therefore, prudence should be exercised when omitting $J \geq 5 / 2 \Lambda^{*}$-resonances in an isobar description of the $p\left(\gamma^{(*)}, K\right) \Lambda$ process.

The results for the photo-amplitudes are summarized in table 1. This table is useful for any isobar model involving real photons which couple to a $\Lambda^{*}$-resonance. Experimental numbers for the EM decay of $\Lambda^{*}$ 's are rare. Essentially, only the decay widths for the two lowest-lying $\Lambda$-resonances are known. Of these two, the $S_{01}(1405)$ is often suggested to have a peculiar structure, which falls beyond the scope of CQ model calculations $[32,33]$. In view of the computed value for the EM decay width largely overshooting the measured one, our calculations seem to confirm this conjecture. The properties of the $D_{03}(1520)$ are, however, reasonably well reproduced. Table 1 also shows that the sole resonances for which PDG mentions an EM decay width, emerge in our calculations indeed with the highest $\Lambda^{*} \rightarrow \Lambda$ widths.

More experimental information on the EM properties of the higher-lying $\Lambda$-resonances would enable us to draw further conclusions about the quality of our calculations. An analysis of $p\left(\gamma^{*}, K\right) Y$ data based on input parameters from our CQ model would be an indirect but stringent test of our model assumptions. At this point, we want to stress again that we have not introduced any new parameters in 

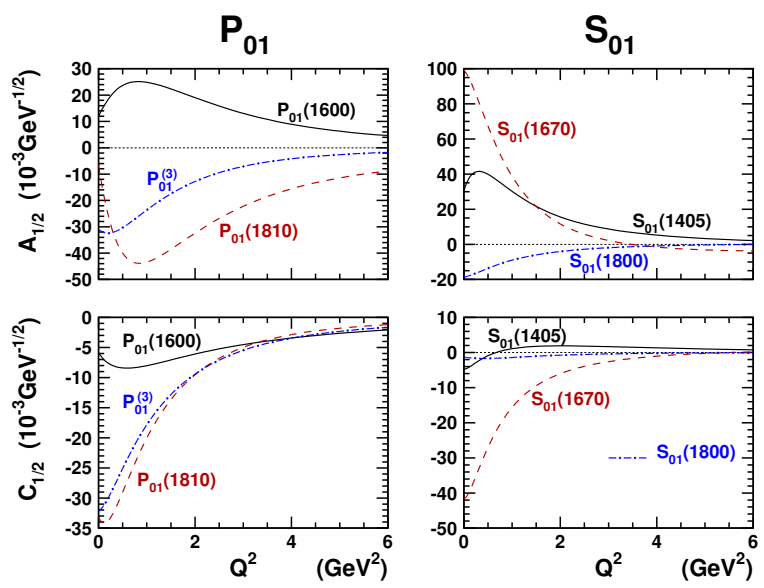

Fig. 10. The $Q^{2}$-dependence for the $\Lambda^{*}+\gamma^{*} \rightarrow \Sigma$ decays for spin $J=1 / 2$ resonances: left (right) panels show the results for the positive (negative)-parity $\Lambda^{*}$-resonances.

the current operator, which makes our results parameterfree predictions.

\section{$4.2 \Lambda^{*}+\gamma^{(*)} \rightarrow \boldsymbol{\Sigma}$ transitions}

Investigations of the $\gamma^{(*)}+p \rightarrow K^{+}+\Sigma^{0}$ reaction in ref. [2] have indicated that a proper modeling of the background terms requires the introduction of hyperon resonances with isospin $T=0$ and $T=1$, i.e. $\Lambda$ as well as $\Sigma$-resonances.

In figs. 10 and 11, we display the HAs for the spin $J=1 / 2$ and $J=3 / 2$ resonances, respectively. Again, one observes that some HAs reach a maximum at moderate values for the momentum transfer squared $\left(Q^{2}<\right.$ $\left.1.5 \mathrm{GeV}^{2}\right)$. This maximum is particularly pronounced for the $A_{1 / 2}$ of the $P_{01}(1810)$ and $D_{03}(1520)$ resonances. For these states, the HA at its maximum is more than double the value at $Q^{2}=0$.

In table 2 , the results for the electromagnetic $\Lambda^{*}(J=$ $1 / 2,3 / 2) \rightarrow \Sigma^{0}(1193)$ decays are summarized for $Q^{2}=0$. The EM decay width for $S_{01}(1405) \rightarrow \Sigma^{0}(1193)$ is clearly overestimated. This is similar to the $S_{01}(1405) \rightarrow \Lambda$ result of table 1 , and could be attributed to the peculiar structure of this resonance. The predicted decay width of the $D_{03}(1520)$ is about a factor of 2 smaller than the experimentally determined value. This is in contrast with the $D_{03}(1520) \rightarrow \Lambda$ transition of table 1 , where the width is overestimated by about a factor of 2 . The discrepancy between computed and measured values might be attributed to the $D_{03}(1520) \rightarrow \bar{K} N \rightarrow \gamma Y$ two-step process, which may interfere destructively with the direct $D_{03}(1520) \rightarrow$ $\gamma Y$ process if $Y=\Lambda$ and constructively if $Y=\Sigma^{0}$.

The computed EM decay width of $3.827 \mathrm{MeV}$ for the $S_{01}(1670)$ is exceptionally large. It represents about $10 \%$ of the reported total decay width $\Gamma_{\exp }^{\text {tot }}=25-50 \mathrm{MeV}$ [24]. The Crystal Ball Collaboration at Brookhaven has investigated $\bar{K}^{-} p$ scattering up to $\underline{W}_{-} \sim 1680 \mathrm{MeV}$ [37], and report a cross-section for the $\bar{K}^{-} p \rightarrow \gamma \Sigma^{0}$ reaction
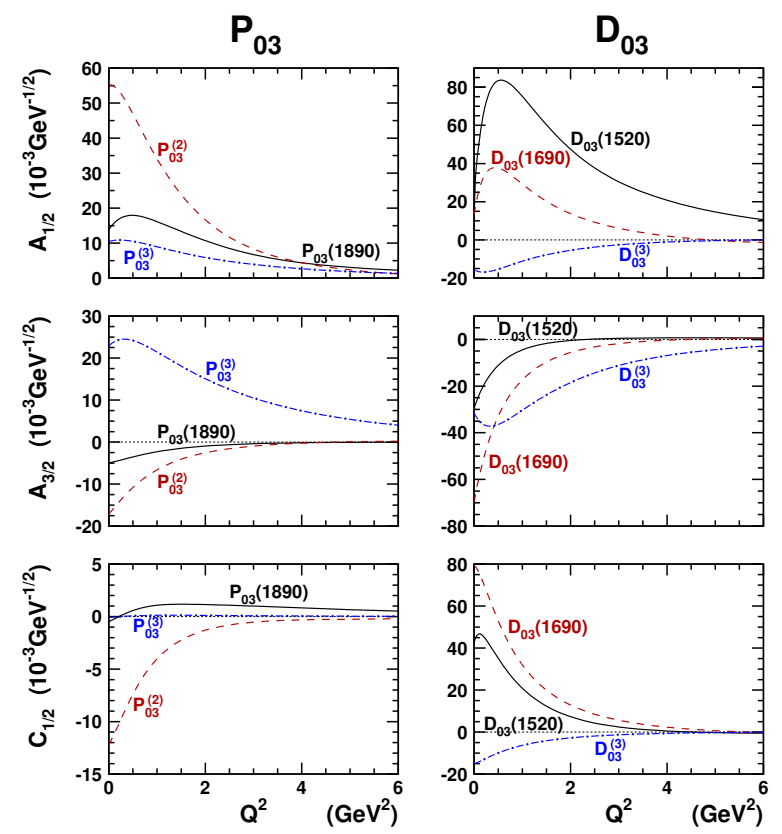

Fig. 11. The $Q^{2}$-dependence for the $\Lambda^{*}+\gamma^{*} \rightarrow \Sigma$ decays for spin $J=3 / 2$ resonances: left (right) panels show the results for the positive (negative)-parity $\Lambda^{*}$-resonances.

Table 2. Calculated masses, photo-amplitudes and EM decay widths for the $\Lambda^{*}+\gamma \rightarrow \Sigma^{0}$ (1193) transition are given below. Values between brackets denote the experimental EM decay width of the $\Lambda$-resonance to the $\Sigma^{0}(1193)$ as given by ref. [24]. Masses and decay widths are expressed in units of $\mathrm{MeV}$, photoamplitudes are given in units of $10^{-3} \mathrm{GeV}^{-1 / 2}$. Note that some masses differ from the values given in table 1 because they were computed in a larger basis.

\begin{tabular}{|c|c|c|c|c|}
\hline Resonance & $M_{\text {calc }}$ & $A_{1 / 2}$ & $A_{3 / 2}$ & $\Gamma_{\text {calc }}$ \\
\hline \hline$P_{01}(1600)$ & 1713 & 12.0 & - & 0.0679 \\
$P_{01}(1810)$ & 1771 & -6.62 & - & 0.0240 \\
$P_{01}^{(3)}$ & 1928 & -30.9 & - & 0.727 \\
$S_{01}(1405)$ & 1538 & 30.3 & - & 0.233 \\
& & & & $(0.010 \pm 0.004 /$ \\
& & & & $0.023 \pm 0.007)$ \\
$S_{01}(1670)$ & 1649 & 99.2 & - & 3.827 \\
$S_{01}(1800)$ & 1855 & -18.7 & - & 0.231 \\
\hline$P_{03}(1890)$ & 1834 & 13.8 & -4.94 & 0.068 \\
$P_{03}^{(2)}$ & 1970 & 55.2 & -17.1 & 1.367 \\
$P_{03}^{(3)}$ & 2068 & 10.5 & 22.9 & 0.303 \\
$D_{03}(1520)$ & 1506 & 23.3 & -30.0 & 0.157 \\
& & & & $\left(0.304_{-0.070}^{+0.076}\right)$ \\
$D_{03}(1690)$ & 1668 & 13.3 & -70.0 & 1.049 \\
$D_{03}^{(3)}$ & 1790 & -15.6 & -31.4 & 0.353 \\
\hline
\end{tabular}

$\sigma_{\text {tot }}=134 \mu \mathrm{b}$ at a kaon lab momentum $p_{K}^{\text {lab }}=750 \mathrm{MeV}$ $(W=1677 \mathrm{MeV})$. This is roughly four times as large as the cross-section for the $\bar{K}^{-} p \rightarrow \gamma \Lambda$ reaction $\left(\sigma_{\text {tot }}=\right.$ $31 \mu \mathrm{b})$. This observation is consistent with the calculated EM decay width for the $S_{01}(1670) \rightarrow \Sigma^{0}$ transition being much larger than for the $S_{01}(1670) \rightarrow \Lambda$ process. 

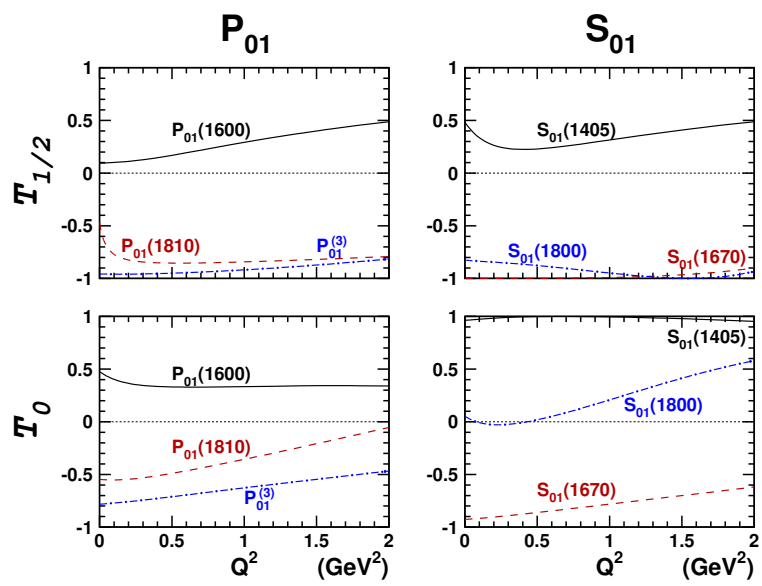

Fig. 12. The asymmetries for $0<Q^{2}<2.0 \mathrm{GeV}^{2}$ as defined in eqs. (26) for the EM decays to different isospin channels for the three lowest-lying $(J=1 / 2, S=-1, I=0) \Lambda^{*}$-resonances with positive parity (left panels) and negative parity (right panels).

The $\bar{K}^{-} p \rightarrow \eta \Lambda$ cross-section at an invariant mass around $1670 \mathrm{MeV}$ was analysed in ref. [38], using six coupled channels $\left(\bar{K} N, \eta \Lambda, \pi \Sigma, \pi \Sigma^{*}(1385), \pi \pi \Lambda\right.$, and $\left.\pi \pi \Sigma\right)$. A partial decay width of $3.6 \pm 1.4 \mathrm{MeV}$ for the $S_{01}(1670) \rightarrow$ $\eta \Lambda$ process was reported. This is comparable in magnitude to the computed EM decay width in table 2. Therefore, including the $\gamma \Sigma$-channel in a coupled-channel analysis of $\bar{K}-p$ scattering at $p_{K} \approx 750 \mathrm{MeV}$ seems relevant.

For the $\Lambda^{*} \rightarrow \Sigma^{0}$ transitions, the second and the third resonances have larger HAs than the first one. Furthermore, the helicity amplitudes at small $Q^{2}$ are quite large. The difference between $\Lambda^{*} \rightarrow \Lambda$ and $\Lambda^{*} \rightarrow \Sigma^{0}$ EM decays can be made more explicit through introducing the following isospin asymmetries:

$$
\begin{gathered}
\mathcal{T}_{1 / 2}=\frac{\left|A_{1 / 2}^{\Lambda}\right|^{2}-\left|A_{1 / 2}^{\Sigma}\right|^{2}}{\left|A_{1 / 2}^{\Lambda}\right|^{2}+\left|A_{1 / 2}^{\Sigma}\right|^{2}}, \\
\mathcal{T}_{3 / 2}=\frac{\left|A_{3 / 2}^{\Lambda}\right|^{2}-\left|A_{3 / 2}^{\Sigma}\right|^{2}}{\left|A_{3 / 2}^{\Lambda}\right|^{2}+\left|A_{3 / 2}^{\Sigma}\right|^{2}}, \\
\mathcal{T}_{0}=\frac{\left|C_{1 / 2}^{\Lambda}\right|^{2}-\left|C_{1 / 2}^{\Sigma}\right|^{2}}{\left|C_{1 / 2}^{\Lambda}\right|^{2}+\left|C_{1 / 2}^{\Sigma}\right|^{2}} .
\end{gathered}
$$

Here, the superscript $\Lambda\left(\Sigma^{0}\right)$ stands for the decay of the resonance to the $\Lambda\left(\Sigma^{0}\right)$ ground state. It is clear that a positive (negative) value indicates that the resonance will preferentially decay to the $\Lambda\left(\Sigma^{0}\right)$ ground state. As can be inferred from figs. 12 and 13, the first resonance generally has positive isospin asymmetries, while the higher-lying resonances mostly have negative isospin asymmetries at low momentum transfer squared $\left(Q^{2}<2.0 \mathrm{GeV}^{2}\right)$.

At this point, it is convenient to compare our results with previous investigations of EM decay widths of $\Lambda^{*}$-resonances. To our knowledge, these are limited to
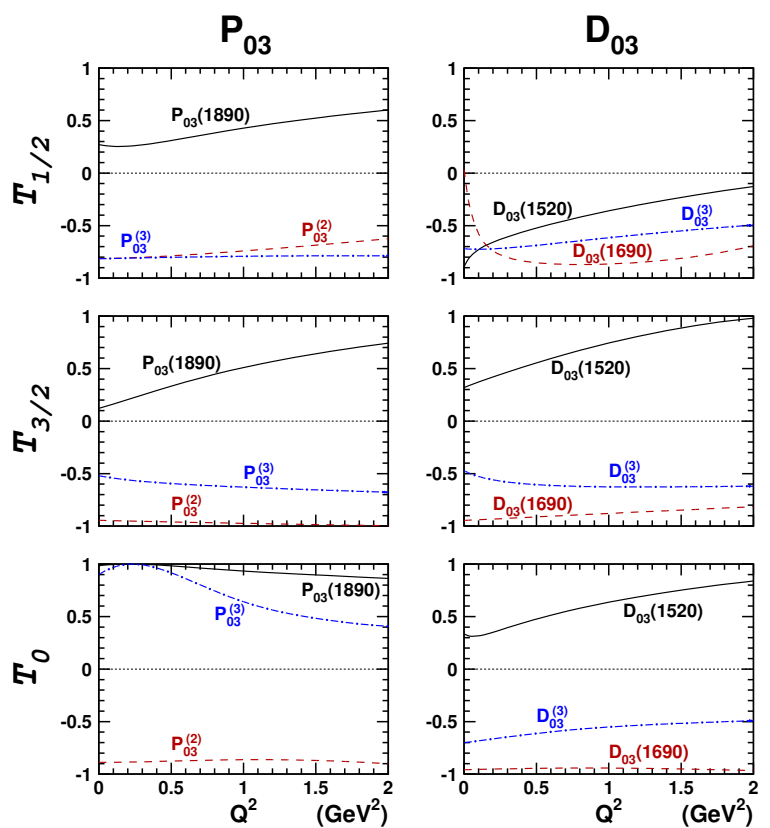

Fig. 13. The asymmetries for $0<Q^{2}<2.0 \mathrm{GeV}^{2}$ as defined in eqs. (26) for the EM decays to different isospin channels for the three lowest-lying $(J=3 / 2, S=-1, I=0) \Lambda^{*}$-resonances with positive parity (left panels) and negative parity (right panels).

Table 3. Comparison of the EM decay widths (in $\mathrm{keV}$ ) of the $S_{01}(1405)$ and $D_{03}(1520)$ hyperon resonances with previous calculations from refs. [12] (DHK83), [13] (KMS85), [14] (WPR91), [15] (UM93), and [19] (SSG95). The experi-

\begin{tabular}{|c|c|c|c|c|}
\hline 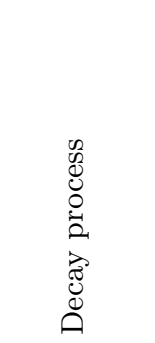 & 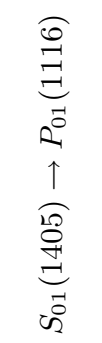 & 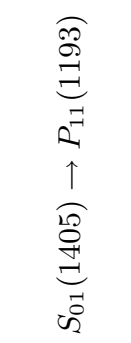 & 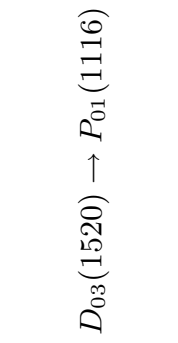 & 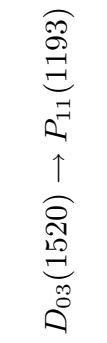 \\
\hline DHK83 & 143 & 91 & 96 & 74 \\
\hline $\begin{array}{r}\text { KMS85 I } \\
\text { II }\end{array}$ & $\begin{array}{l}60 \\
17\end{array}$ & $\begin{array}{l}18 \\
2.7\end{array}$ & $\begin{array}{l}46 \\
27\end{array}$ & $\begin{array}{c}17 \\
102\end{array}$ \\
\hline WPR91 & 118 & 46 & 215 & 293 \\
\hline UM93 & 31.46 & 50.85 & 74.98 & 1.85 \\
\hline $\begin{array}{r}\text { SSG95 Ia } \\
\text { Ib } \\
\text { IIa } \\
\text { IIb }\end{array}$ & $\begin{array}{l}67 \\
44 \\
56 \\
40\end{array}$ & $\begin{array}{l}29 \\
13 \\
29 \\
17\end{array}$ & $\begin{array}{l}- \\
- \\
- \\
-\end{array}$ & $\begin{array}{l}- \\
- \\
- \\
-\end{array}$ \\
\hline This work & 912 & 233 & 258 & 157 \\
\hline $\begin{array}{c}\text { PDG } \\
\text { CLAS05 }\end{array}$ & $\begin{array}{c}27 \pm 8 \\
-\end{array}$ & $\begin{array}{c}10 \pm 4 / \\
23 \pm 7 \\
-\end{array}$ & $\begin{array}{c}125_{-38}^{+42} \\
167 \pm 43_{-12}^{+26}\end{array}$ & $304_{-70}^{+76}$ \\
\hline
\end{tabular}
mental values in the bottom rows are taken from the PDG [24] and from ref. [39] (CLAS05). 
the $S_{01}(1405)$ and $D_{03}(1520)$ resonances. Table 3 displays the model results reported in refs. $[12-15,19]$, as well as our own findings and the experimental values from the PDG [24] and from CLAS [39]. It is quite striking that the models KMS85 [13], UM93 [15] and SSG95 [19], which put the nonstrange quark mass to zero (chiral limit), obtain qualitative good results for the $S_{01}(1405)$, but fall short in their description of the $D_{03}(1520)$. In our framework, the decay widths of the $S_{01}(1405)$ are roughly an order of magnitude larger than for the other models, but the predictions for the $D_{03}(1520)$-resonance are in qualitative agreement with the measured decay widths. Also in model WPR91 [14], the EM decay widths of the $S_{01}(1405)$ are overestimated, while the ones for the $D_{03}(1520)$ are in very good agreement with experiment. We have already argued that our model assumptions are inadequate to describe the $S_{01}(1405)$-resonance. Clearly, the key for understanding this resonance should be sought in other approaches to baryon structure. For the $D_{03}(1520)$, the comparison of our model with the "chiral" models and with the nonrelativistic CQ model DHK83 [12] seems to indicate that this resonance is well-described by a bound state of three relativistic constituent quarks.

\section{Results for the $\Sigma$-resonances}

\section{$5.1 \Sigma^{*}+\gamma^{(*)} \rightarrow \Lambda$ transitions}

In this section we will discuss the EM helicity amplitudes of the $\Sigma^{* 0}(J=1 / 2,3 / 2) \rightarrow \Lambda$ process. The experimental situation for the $\Sigma$ spectrum is even worse than for the $\Lambda$. Except for the octet $\Sigma(1193)$ and the decuplet $\Sigma^{*}(1385)$, only four 4-star and four 3-star resonances are reported in ref. [24], and of these, the spin and parity are unknown for the $\Sigma(2250)$. Furthermore, to our knowledge there are no data with regard to the EM properties of these resonances.

The predictions from the Bethe-Salpeter model for the photo-amplitudes and EM decay widths are presented in table 4 for the $J=1 / 2$ (top rows) and $J=3 / 2$ (bottom rows) $\Sigma^{*}$-resonances. The three lowest $\Sigma^{*}$ 's with $J=1 / 2$ from our calculations are referred to as $P_{11}(1660), P_{11}^{(2)}$ and $P_{11}^{(3)}$. The existence of the $P_{11}(1770)$ is based on one analysis, and is questionable [24]. Therefore, it is argued in ref. [31] that the $P_{11}(1770)$ should be disregarded, and that the $P_{11}(1880)$ is actually the second-lowest resonance $P_{11}^{(2)}$. Even then, the predicted masses are about $100 \mathrm{MeV}$ too high. For the negative-parity $\Sigma^{*}$-resonances, the situation for the $J=1 / 2$ resonances is clearer. The identification of the two lowest-lying computed states with the experimentally observed ones is straightforward by comparing the measured and the predicted masses. The $S_{11}(2000)$ can be identified with the third computed state, since the value of $2000 \mathrm{MeV}$ for its experimental mass is a very rough estimate [24]. The computed EM decay widths in table 4 decrease with increasing mass for the $P_{11}$ as well as for the $S_{11}$-resonances.

Table 4 also shows the EM decay widths of the lowest-lying $J=3 / 2 \Sigma$-resonances. One clearly observes
Table 4. Calculated masses, photo-amplitudes and EM decay widths for the $\Sigma^{* 0}+\gamma \rightarrow \Lambda(1116)$ transitions for the lowestlying $J=1 / 2$ (top rows) and $J=3 / 2$ (bottom rows) resonances. Masses and decay widths are given in units of $\mathrm{MeV}$, photo-amplitudes are given in units of $10^{-3} \mathrm{GeV}^{-1 / 2}$. In the last column, the value between brackets denotes the experimental upper limit of the EM decay width of the $\Sigma$-resonance to the $\Lambda(1116)$ as given by ref. [24].

\begin{tabular}{|c|c|c|c|c|}
\hline Resonance & $M_{\text {calc }}$ & $A_{1 / 2}$ & $A_{3 / 2}$ & $\Gamma_{\text {calc }}$ \\
\hline \hline$P_{11}(1660)$ & 1801 & 26.1 & - & 0.451 \\
$P_{11}^{(2)}$ & 1967 & -15.7 & - & 0.216 \\
$P_{11}^{(3)}$ & 2049 & 5.47 & - & 0.0294 \\
$S_{11}(1620)$ & 1640 & 58.2 & - & 1.551 \\
$S_{11}(1750)$ & 1800 & 24.7 & - & 0.403 \\
$S_{11}(2000)$ & 1813 & -16.9 & - & 0.193 \\
\hline$P_{13}(1385)$ & 1409 & 63.7 & 109.8 & 1.527 \\
& & & & $(<13.94)$ \\
$P_{13}(1840)$ & 1902 & -29.4 & 9.56 & 0.378 \\
$P_{13}(2080)$ & 1950 & 26.6 & 44.7 & 1.155 \\
$D_{13}(1580)$ & 1675 & 14.2 & -36.8 & 0.390 \\
$D_{13}(1670)$ & 1727 & 36.1 & 61.9 & 1.457 \\
$D_{13}^{(3)}$ & 1780 & -38.1 & -27.8 & 0.706 \\
\hline
\end{tabular}
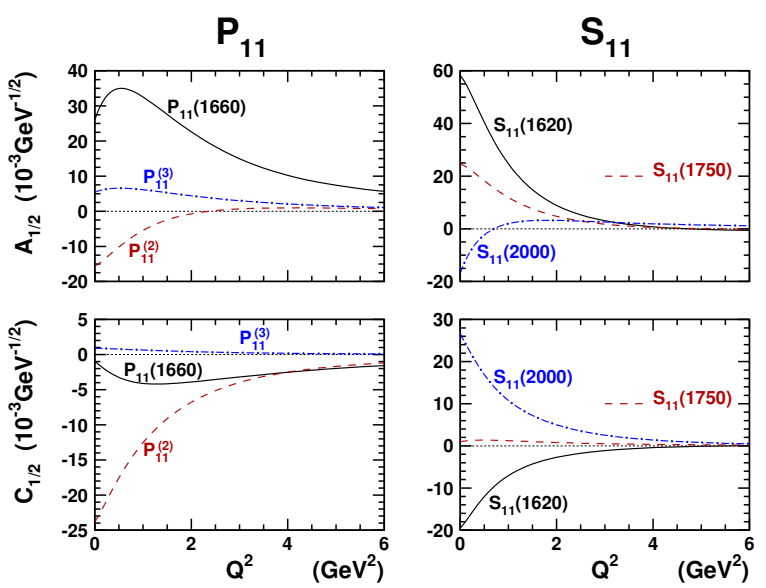

Fig. 14. The $Q^{2}$-dependence for the $\Sigma^{* 0}+\gamma^{*} \rightarrow \Lambda$ decays for spin $J=1 / 2$ resonances: left (right) panels show the results for the positive (negative)-parity $\Sigma^{*}$-resonances.

rather large values for the $P_{13}(1385)$, the $P_{13}(2080)$ and the $D_{13}(1670)$ resonances. The first is a member of the baryon decuplet. The PDG provides only a rough upper limit around $13.94 \mathrm{MeV}$ for the EM decay width of the $\Sigma^{*}(1385)$ to the $\Lambda(1116)$ [24]. Our computed value is well below that limit. The $D_{13}(1670)$-resonance could magnify the effect of the $S_{01}(1670)$ in the $\bar{K}^{-} p \rightarrow \gamma \Lambda$ process, increasing the total cross-section of the latter reaction even more at $W \approx 1670 \mathrm{MeV}$.

For the $P_{11}$-resonances, the results for the HAs are displayed in the left panels of fig. 14. The $P_{11}(1660)$, which is the analogue of the Roper resonance in the $\Sigma$ spectrum, has the largest $A_{1 / 2}$, reaching a maximum at $Q^{2} \approx 0.5 \mathrm{GeV}^{2}$. The second resonance has the largest 

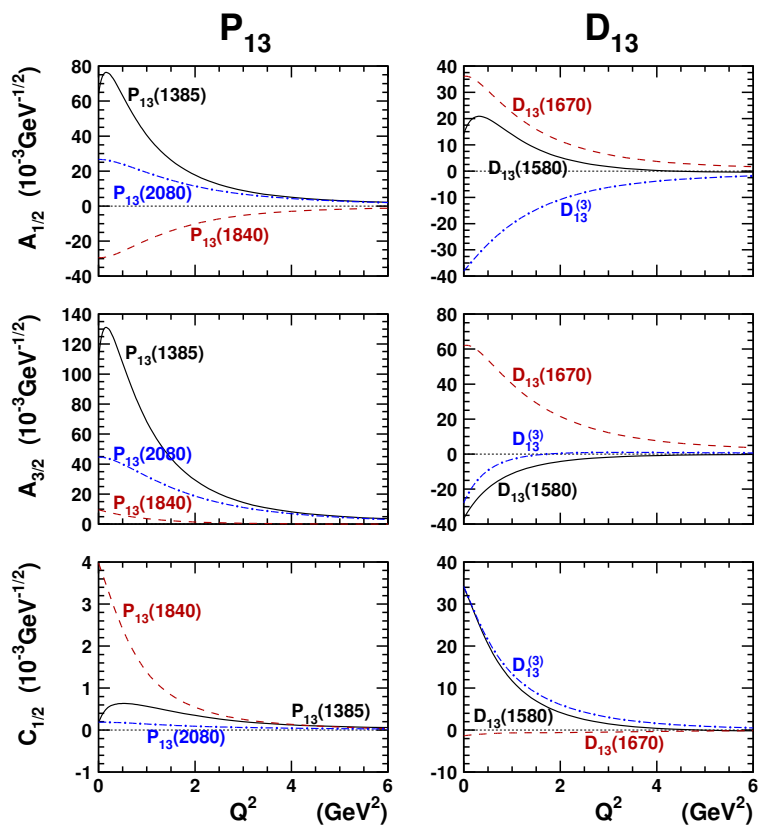

Fig. 15. The $Q^{2}$-dependence for the $\Sigma^{* 0}+\gamma^{*} \rightarrow \Lambda$ decays for spin $J=3 / 2$ resonances: left (right) panels show the results for the positive (negative)-parity $\Sigma^{*}$-resonances.

$C_{1 / 2}$ for small to moderate $Q^{2}$-values. The HAs for the $P_{11} \Sigma^{*} \rightarrow \Lambda$ decays are comparable to those for the $P_{01} \Lambda^{*} \rightarrow \Lambda$ decays. Therefore, the $\Sigma^{*}$ 's can be expected to contribute significantly to the background of the $p\left(\gamma^{(*)}, K\right) \Lambda$ and the $\bar{K}^{-} p \rightarrow \gamma \Lambda$ cross-sections. This observation is even more relevant to the $J^{\pi}=1 / 2^{-}$resonances, for which the HAs are depicted in the right panels of fig. 14. One observes a large $A_{1 / 2}$ for the $S_{11}(1620)$, which is a 2-star resonance in ref. [24]. This is also clear from the large EM decay width of this resonance, reported in table 4 . However, the data for the $\bar{K}^{-} p \rightarrow \gamma \Lambda$ process [40], do not show a significant enhancement at $W \approx 1620 \mathrm{MeV}$ $\left(p_{K} \approx 629 \mathrm{MeV}\right)$. This could be explained by a small coupling of the $S_{11}(1620)$-resonance to the $\bar{K} N$-channel.

The computed helicity amplitudes for the $J=3 / 2$ $\Sigma^{*}$ 's are displayed in fig. 15 . The decuplet member $P_{13}(1385)$ has the largest HAs of the positive-parity resonances. The $C_{1 / 2}$ 's of the three lowest $P_{13}$ states are all rather small. The helicity amplitudes of the three lowestlying negative-parity $\Sigma$-resonances are moderate, except for the $C_{1 / 2}$ of the $D_{13}(1670)$, which practically vanishes.

\section{$5.2 \Sigma^{*}+\gamma^{(*)} \rightarrow \boldsymbol{\Sigma}$ transitions}

The $\Sigma^{*}+\gamma^{(*)} \rightarrow \Sigma(1193)$ process differs from the ones of previous sections in that it comes in three versions, one for each member of the $\Sigma^{*}$ isospin triplet. Their EM properties are not independent, however, because of the presumed isospin symmetry of the interactions in the Bonn model ( $u$ - and $d$-quark have the same mass and the effective interactions do not depend on the third component of the isospin quantum number $I_{z}$ of the quark). Knowledge
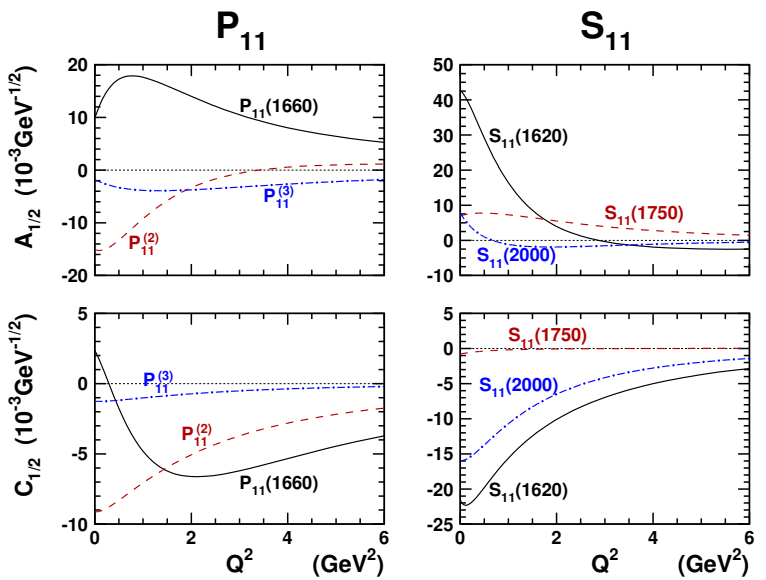

Fig. 16. The $Q^{2}$-dependence for the $\Sigma^{* 0}+\gamma^{*} \rightarrow \Sigma^{0}$ decays for spin $J=1 / 2$ resonances: left (right) panels show the results for the positive (negative)-parity $\Sigma^{*}$-resonances.

Table 5. Calculated masses, photo-amplitudes and EM decay widths for the $\Sigma^{*}+\gamma \rightarrow \Sigma(1193)$ transitions for the $J=1 / 2$ $\Sigma^{*}$-resonances. The charge of the $\Sigma^{*}$ isospin triplet member is indicated by the superscript ${ }^{(0,+,-)}$. Masses and decay widths are given in units of $\mathrm{MeV}$, photo-amplitudes are given in units of $10^{-3} \mathrm{GeV}^{-1 / 2}$.

\begin{tabular}{|c|c|c|c|}
\hline Resonance & $M_{\text {calc }}$ & $A_{1 / 2}$ & $\Gamma_{\text {calc }}$ \\
\hline \hline$P_{11}^{0}(1660)$ & 1801 & 9.91 & 0.0578 \\
$P_{11}^{(2) 0}$ & 1967 & -15.1 & 0.186 \\
$P_{11}^{(3) 0}$ & 2049 & -1.83 & 0.00311 \\
$S_{11}^{0}(1620)$ & 1640 & 42.7 & 0.688 \\
$S_{11}^{0}(1750)$ & 1800 & 6.96 & 0.0284 \\
$S_{11}^{0}(2000)$ & 1813 & 7.86 & 0.0373 \\
\hline$P_{11}^{+}(1660)$ & 1801 & 35.3 & 0.733 \\
$P_{11}^{(2)+}$ & 1967 & -54.8 & 2.446 \\
$P_{11}^{(3)+}$ & 2049 & -4.86 & 0.0219 \\
$S_{11}^{+}(1620)$ & 1640 & 125.6 & 5.955 \\
$S_{11}^{+}(1750)$ & 1800 & 4.80 & 0.0135 \\
$S_{11}^{+}(2000)$ & 1813 & 10.3 & 0.0641 \\
\hline$P_{11}^{-}(1660)$ & 1801 & -15.5 & 0.141 \\
$P_{11}^{(2)-}$ & 1967 & 24.6 & 0.493 \\
$P_{11}^{(3)-}$ & 2049 & 1.20 & 0.00136 \\
$S_{11}^{-}(1620)$ & 1640 & -40.3 & 0.613 \\
$S_{11}^{-}(1750)$ & 1800 & 9.12 & 0.0488 \\
$S_{11}^{-}(2000)$ & 1813 & 5.41 & 0.0177 \\
\hline
\end{tabular}

of the helicity amplitudes for the $\Sigma^{*+}$ and the $\Sigma^{*-}$ allows one to obtain those for the $\Sigma^{* 0}$, simply by taking the average. In the following, results for all three isospin triplet members will be presented. The charge of the particle will be denoted in the superscript.

In fig. 16, the HAs for the $\Sigma^{* 0} \rightarrow \Sigma^{0}$ decays are displayed for the lowest-lying spin $J=1 / 2$ resonances. Obviously, the HAs for the $P_{11}^{0}$-resonances are relatively small. This is reflected in the rather small values for the computed EM decay widths of the $P_{11}^{0}$-resonances given 

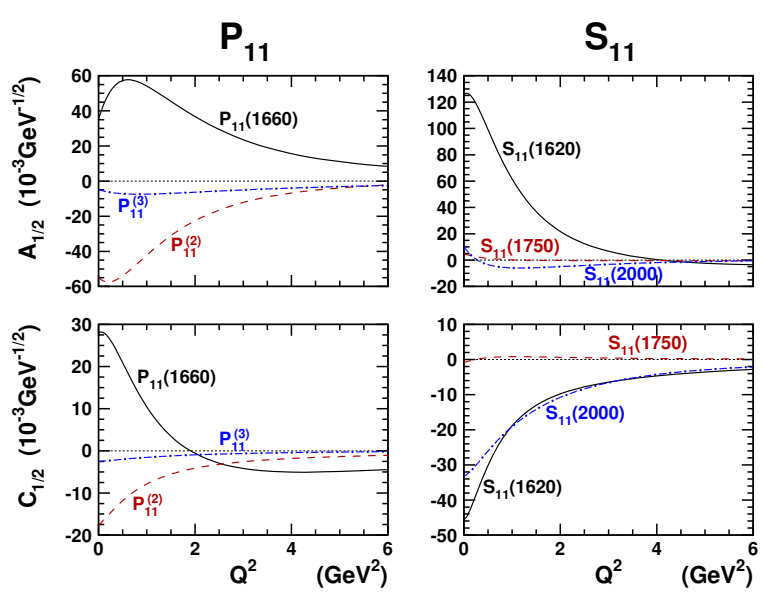

Fig. 17. The $Q^{2}$-dependence for the $\Sigma^{*+}+\gamma^{*} \rightarrow \Sigma^{+}$decays for spin $J=1 / 2$ resonances: left (right) panels show the results for the positive (negative)-parity $\Sigma^{*}$-resonances.
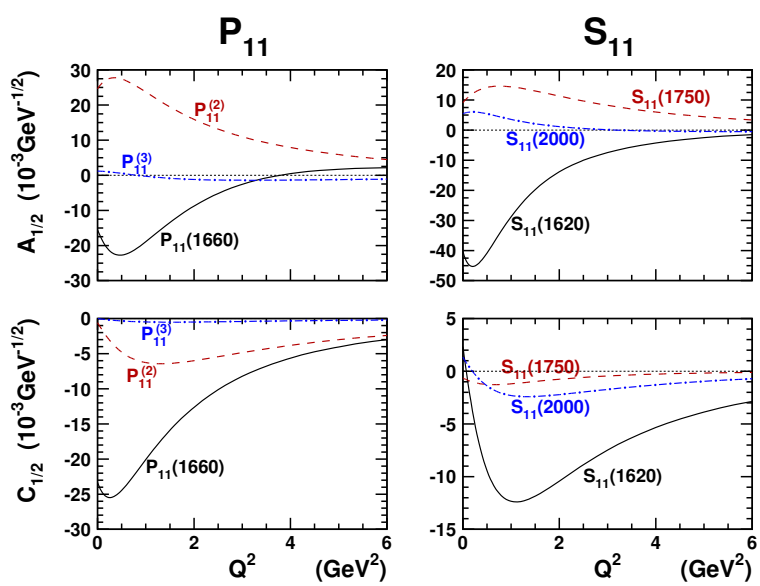

Fig. 18. The $Q^{2}$-dependence for the $\Sigma^{*-}+\gamma^{*} \rightarrow \Sigma^{-}$decays for spin $J=1 / 2$ resonances: left (right) panels show the results for the positive (negative)-parity $\Sigma^{*}$-resonances.

in table 5. A larger EM response is seen for the negativeparity states, where the $S_{11}^{0}(1620)$ has HAs of similar magnitude as the ones for the decay to the $\Lambda(1116)$ (fig. 14). The other $S_{11}^{0}$-resonances have rather small HAs.

The results for the positively charged members of the $\Sigma^{*}$ triplets, which are presented in fig. 17 , are quite surprising. In contrast with their neutral counterparts, the first and second $P_{11}^{+}$-resonances have large helicity amplitudes. This can also be deduced from the predictions for the EM decay widths in table 5. These findings have serious implications when modeling the background contributions in $p\left(\gamma^{(*)}, K\right) Y$ processes. When $Y$ is a neutral hyperon $\left(\Lambda\right.$ or $\left.\Sigma^{0}\right)$, the exchanged particle in the $u$-channel (fig. 1) would necessarily be neutral. The $P_{11}^{0}$-resonances are likely to have a negligible effect because of their small EM couplings. When $Y=\Sigma^{+}$, the intermediate hyperon would be positively charged, and the $P_{11}^{+}$-resonances could contribute sizably to the background.

For the $S_{11}^{+}$-resonances, a striking feature is the large EM decay width of the first resonance in table 5. Again,
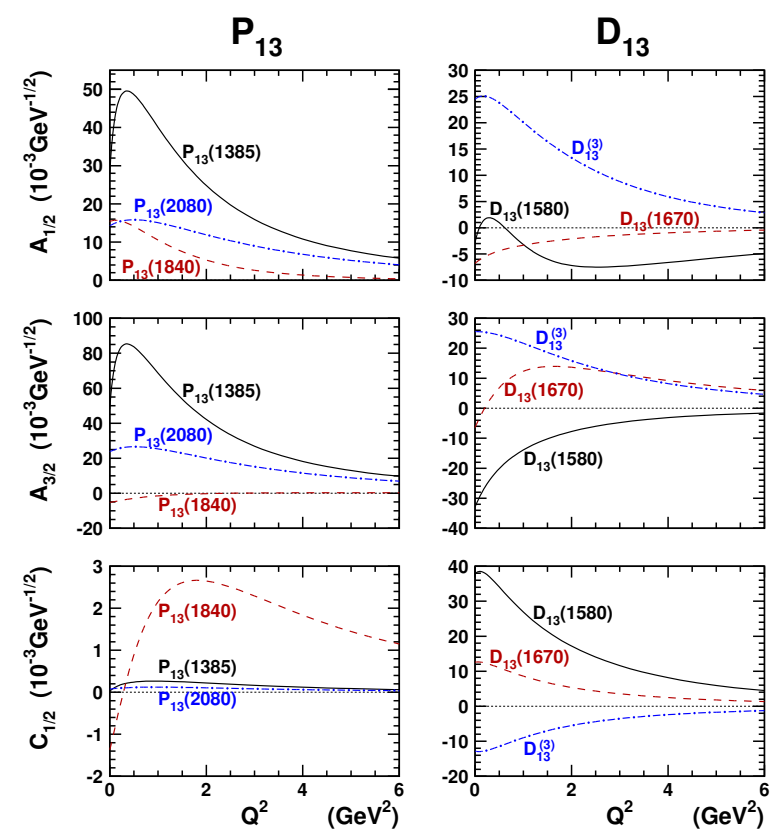

Fig. 19. The $Q^{2}$-dependence for the $\Sigma^{* 0}+\gamma^{*} \rightarrow \Sigma^{0}$ decays for spin $J=3 / 2$ resonances: left (right) panels show the results for the positive (negative)-parity $\Sigma^{*}$-resonances.

this indicates the large coupling of the $S_{11}^{+}(1620)$ to the $\gamma Y$ decay channels. Furthermore, the EM decay width $\Gamma_{\text {calc }}^{\mathrm{EM}} \simeq 6 \mathrm{MeV}$ seems to be a significant fraction of the poorly known total decay width $\Gamma_{\text {exp }}^{\text {tot }}=10-106 \mathrm{MeV}[24]$. Since the latter was extracted from meson-baryon scattering experiments, it is possible that the experimental status of this resonance can be improved considerably by investigating radiative processes. The computed HAs of the other $S_{11}^{+}$-resonances again turn out to be relatively small.

The calculated HAs for the $P_{11}^{-}$and $S_{11}^{-} \Sigma^{*}$-resonances are displayed in fig. 18. Moderate HAs and EM decay widths (table 5) are observed for the positive-parity resonances. For the negative-parity resonances, one notices the large $A_{1 / 2}$ for the $S_{11}^{-}(1620)$-resonance.

The HAs for the neutral process $\Sigma^{* 0}+\gamma^{*} \rightarrow \Sigma^{0}$ are shown in fig. 19 for $J=3 / 2$ resonances. For the $P_{13}$-resonances, one can point to the relatively large $A_{1 / 2}$ and $A_{3 / 2}$ amplitudes for the decuplet member $P_{13}(1385)$. Yet, due to the small phase space, this does not result in a large EM decay width, as presented in table 6 . The $P_{13}$ (2080)-resonance, on the other hand, has only moderately large helicity amplitudes, yet has a larger EM decay width than the $P_{13}(1385)$ due to its larger mass. The results for the negative-parity resonances are displayed in the right panels of fig. 19. There, one notices the small HAs of the $D_{13}(1670)$-resonance. Consequently, this resonance has a small EM decay width (cf. table 6). The computed HAs of the other two $D_{13}$-resonances are of intermediate magnitude.

We also present the results for the HAs of the charged $\Sigma^{*+}+\gamma^{*} \rightarrow \Sigma^{+}$process in fig. 20. This figure shows that resonances for which the HAs of the neutral process were small or moderate, can still have large HAs for the (positively) charged process, as was the case with the 
Table 6. Calculated masses, photo-amplitudes and EM decay widths for the $\Sigma^{*}+\gamma \rightarrow \Sigma(1193)$ transitions for $J=3 / 2$ $\Sigma^{*}$-resonances. The charge of the $\Sigma^{*}$ isospin triplet member is indicated by the superscript ${ }^{(0,+,-)}$. Masses and decay widths are given in units of $\mathrm{MeV}$, photo-amplitudes are given in units of $10^{-3} \mathrm{GeV}^{-1 / 2}$. The value between brackets is the experimental upper limit quoted by the PDG [24], with a $90 \%$ confidence level.

\begin{tabular}{|c|c|c|c|c|}
\hline Resonance & $M_{\text {calc }}$ & $A_{1 / 2}$ & $A_{3 / 2}$ & $\Gamma_{\text {calc }}$ \\
\hline \hline$P_{13}^{0}(1385)$ & 1409 & 27.8 & 48.0 & 0.181 \\
$P_{13}^{0}(1840)$ & 1902 & 15.4 & -5.25 & 0.0960 \\
$P_{13}^{0}(2080)$ & 1950 & 14.3 & 23.7 & 0.303 \\
$D_{13}^{0}(1580)$ & 1675 & -2.82 & -32.9 & 0.230 \\
$D_{13}^{0}(1670)$ & 1727 & -6.77 & -6.45 & 0.0214 \\
$D_{13}^{(3) 0}$ & 1780 & 24.4 & 25.5 & 0.349 \\
\hline$P_{13}^{+}(1385)$ & 1409 & 62.6 & 108.2 & 0.920 \\
$P_{13}^{+}(1840)$ & 1902 & 80.0 & -25.6 & 2.559 \\
$P_{13}^{+}(2080)$ & 1950 & 29.7 & 48.5 & 1.280 \\
$D_{13}^{+}(1580)$ & 1675 & 40.0 & -65.2 & 1.235 \\
$D_{13}^{+}(1670)$ & 1727 & -13.1 & -40.3 & 0.440 \\
$D_{13}^{(3)+}$ & 1780 & 59.8 & 40.8 & 1.468 \\
\hline$P_{13}^{-}(1385)$ & 1409 & -7.06 & -12.2 & 0.0117 \\
$P_{13}^{-}(1840)$ & 1902 & -47.1 & 15.1 & $(<0.0095 \pm 0.0006)$ \\
$P_{13}^{-}(2080)$ & 1950 & -1.20 & -1.05 & 0.887 \\
$D_{13}^{-}(1580)$ & 1675 & -45.7 & -0.588 & 0.441 \\
$D_{13}^{-}(1670)$ & 1727 & -0.397 & 27.4 & 0.184 \\
$D_{13}^{(3)-}$ & 1780 & -10.9 & 10.3 & 0.0630 \\
\hline
\end{tabular}
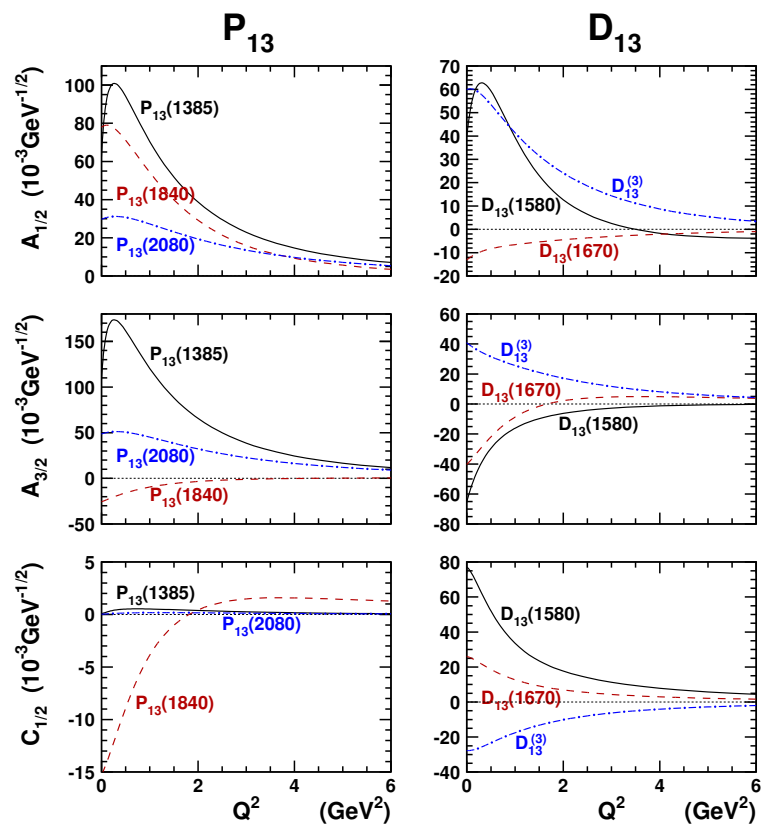

Fig. 20. The $Q^{2}$-dependence for the $\Sigma^{*+}+\gamma^{*} \rightarrow \Sigma^{+}$decays for spin $J=3 / 2$ resonances: left (right) panels show the results for the positive (negative)-parity $\Sigma^{*}$-resonances.
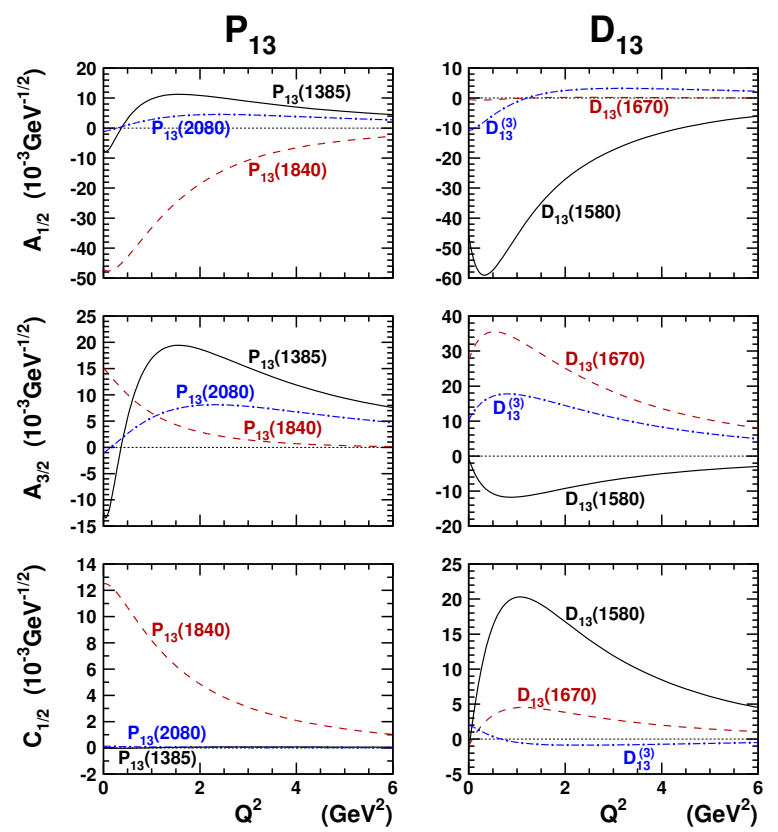

Fig. 21. The $Q^{2}$-dependence for the $\Sigma^{*-}+\gamma^{*} \rightarrow \Sigma^{-}$decays for spin $J=3 / 2$ resonances: left (right) panels show the results for the positive (negative)-parity $\Lambda^{*}$-resonances.

$J=1 / 2 \Sigma$-resonances. This is made even clearer in table 6 , where it is seen that the EM decay widths of the positively charged $\Sigma^{*}$ 's are a factor of 5 or more larger than those of the neutral resonances. This feature is less pronounced for the negatively charged $\Sigma^{*-}+\gamma^{*} \rightarrow \Sigma^{-}$ process. In fig. 21, one does not observe HAs with a magnitude larger than $100 \times 10^{-3} \mathrm{GeV}^{-1 / 2}$. All the computed EM decay widths contained in table 6 are smaller than 1.0 MeV.

The EM properties of the $P_{13}(1385)$-resonance are well investigated in previous articles. In table 7 , we are comparing the EM decay widths from various earlier approaches with ours and with experiment. It is clear that all models predict the EM decay width for the $P_{13}^{0}(1385) \rightarrow$ $P_{01}(1116)$ process within the rough upper limit quoted by the PDG [24]. However, the recent measurement of the CLAS Collaboration [39] sets a more severe limit to this observable. While other models underestimate the newly measured width by roughly a factor of two, our model overestimates the same width by a factor of three. Also, the EM decay width for the $P_{13}^{-}(1385) \rightarrow P_{11}^{-}(1193)$ process is slightly larger in our approach than the rough experimental upper limit. However, all models agree on the relative sizes of the different EM decay widths:

$$
\Gamma_{\gamma \Lambda}>\Gamma_{\gamma \Sigma^{+}}>\Gamma_{\gamma \Sigma^{0}}>\Gamma_{\gamma \Sigma^{-}} .
$$

Furthermore, the vanishing of $C_{1 / 2}$ of the $\Sigma^{*+}(1385)$ in fig. 20 at small $Q^{2}$ (long-wavelength limit) implies that the quadrupole moment related to this transition is very small. If the quadrupole moment is of the same magnitude as the one for the $\Delta^{+}(1232) \rightarrow p(938)$ transition, extra model ingredients leading to larger $D$-wave contributions to the wave functions need to be introduced [41]. 
Table 7. Comparison of the EM decay widths (in $\mathrm{keV})$ of the $P_{11}(1385)$ isotriplet with previous calculations from refs. [12] (DHK83), [13] (KMS85), [14] (WPR91), [16] (LDW93), [18] (SGS95), [20] (AWR96), [22] (WBF00), and [23] (LM04). The experimental values in the bottom rows are taken from the PDG [24] and from ref. [39] (CLAS05).

\begin{tabular}{|c|c|c|c|c|}
\hline 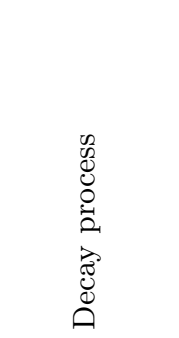 & 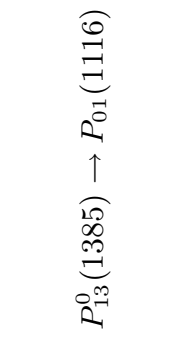 & 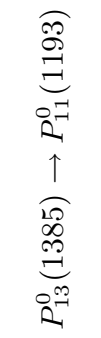 & 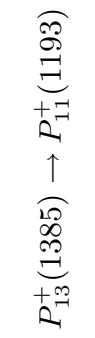 & 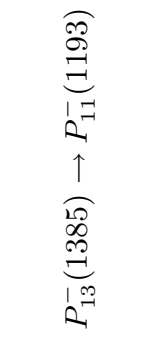 \\
\hline DHK83 & 232 & 19 & 104 & 2.5 \\
\hline KMS85 & 152 & 15 & - & - \\
\hline WPR91 & 267 & 23 & - & - \\
\hline LDW93 & - & 18 & 100 & 2.4 \\
\hline $\begin{array}{r}\text { SGS95 I } \\
\text { II }\end{array}$ & $\begin{array}{l}243 \\
170\end{array}$ & $\begin{array}{l}19 \\
11\end{array}$ & $\begin{array}{l}91 \\
59\end{array}$ & $\begin{array}{l}1 \\
1\end{array}$ \\
\hline $\begin{array}{r}\text { AWR96 Ia } \\
\text { Ib } \\
\text { IIa } \\
\text { IIb }\end{array}$ & $\begin{array}{l}195 \\
180 \\
209 \\
194\end{array}$ & $\begin{array}{l}16 \\
15 \\
12 \\
12\end{array}$ & $\begin{array}{l}81 \\
78 \\
74 \\
71\end{array}$ & $\begin{array}{l}1 \\
1 \\
2 \\
2\end{array}$ \\
\hline WBF00 & 249 & 16.8 & 99 & 3.10 \\
\hline LM04 & $\begin{array}{c}298 \\
( \pm 25)\end{array}$ & $\begin{array}{c}24.9 \\
( \pm 4.1)\end{array}$ & $\begin{array}{c}118 \\
( \pm 10)\end{array}$ & $\begin{array}{c}0.58 \\
( \pm 0.70)\end{array}$ \\
\hline This work & 1527 & 181 & 920 & 11.7 \\
\hline $\begin{array}{c}\text { PDG } \\
\text { CLAS05 }\end{array}$ & $\begin{array}{c}<13940 \\
479 \pm 120^{+81}\end{array}$ & - & - & $\begin{array}{c}<9.5 \pm 0.6 \\
(90 \% \mathrm{CL}) \\
-\end{array}$ \\
\hline
\end{tabular}

Exchange currents [42] or meson loops [43] might be relevant. Therefore, further work needs to be done in order to come to a satisfactory description of the EM properties of the decuplet $\Sigma^{*}$ isotriplet.

\section{Helicity asymmetries}

For hyperon resonances with $J \geq 3 / 2$, the behaviour of the helicity asymmetries can be qualitatively understood. These asymmetries are defined analogous to the isospin asymmetries of eq. (26),

$$
\mathcal{A}=\frac{\left|A_{1 / 2}\right|^{2}-\left|A_{3 / 2}\right|^{2}}{\left|A_{1 / 2}\right|^{2}+\left|A_{3 / 2}\right|^{2}} .
$$

The helicity asymmetries of the lowest-lying $J=3 / 2$ and $J=5 / 2 \Lambda^{*}$-resonances for the decay to the $\Lambda$ ground state are shown in fig. 22 and the asymmetries for the $J=3 / 2$ $\Lambda^{*}$-resonances for the decay to the $\Sigma^{0}$ ground state are
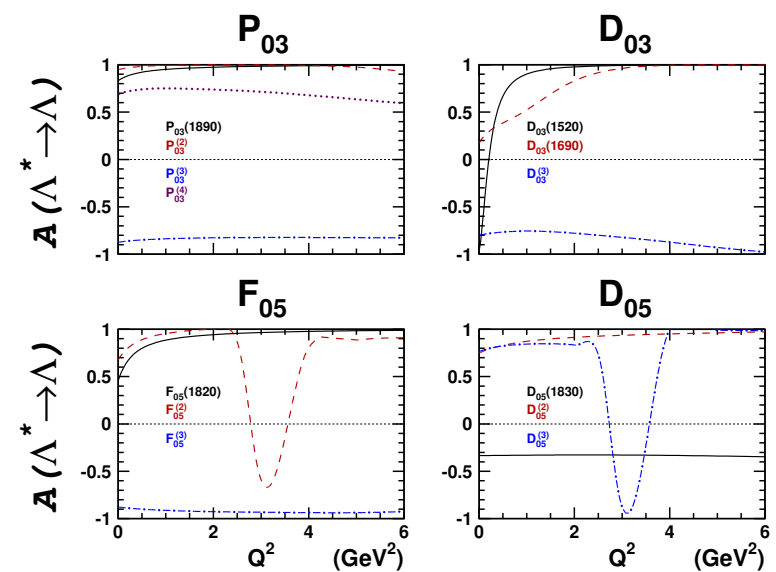

Fig. 22. The helicity asymmetry as defined in eq. (28) for the lowest-lying spin $J=3 / 2$ and $J=5 / 2 \Lambda$-resonances decaying to the $\Lambda$ ground state.
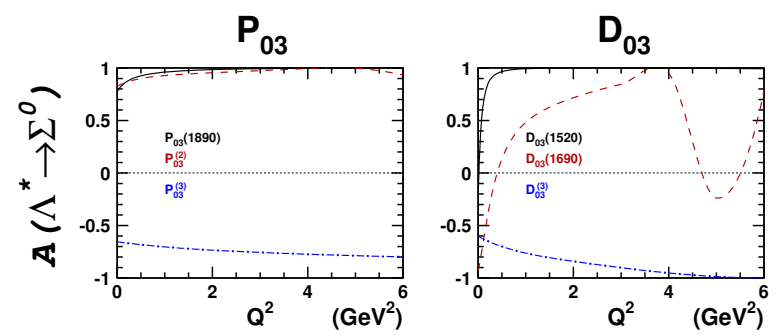

Fig. 23. The helicity asymmetry as defined in eq. (28) for the lowest-lying spin $J=3 / 2$ resonances decaying to the $\Sigma^{0}$ ground state.

displayed in fig. 23. In most cases, the helicity asymmetries approach +1 for high $Q^{2}$, yet for some resonances, the helicity asymmetry is negative.

To understand the sign of the asymmetries, one can project the corresponding BS amplitude on the $S U(6)$ spin-flavour basis states [44]. This was done in ref. [8]. It turns out that the BS amplitudes of the resonances for which $\mathcal{A}$ approaches +1 , receive their largest contribution from $S U(6)$ spin-flavour states for which the total spin $S=1 / 2$. On the other hand, the BS amplitudes of the resonances for which the helicity asymmetry becomes negative at high $Q^{2}$, are dominated by $S=3 / 2 S U(6)$ states.

This observation can be explained qualitatively by considering the EM decay of e.g. a $D_{03}$-resonance to a groundstate hyperon $Y$ in the resonance rest frame (see fig. 24). For high $Q^{2}$, the photon preferentially couples to the individual CQs, which means that the major contribution to the $A_{1 / 2}$ comes from the process in fig. 24(a). There, one of the CQs with negative spin projection along the $z$-axis emits a photon of positive helicity, and flips its spin. This process is allowed for all $D_{03}$-resonances. When the BS amplitude has its main contributions from $S U(6)$ states for which $S=1 / 2$, the major contribution to the $A_{3 / 2}$ comes from the process in fig. 24(b). There, one could argue that the photon is emitted by the resonance as a whole, because the spin projections of the three CQs remain unaltered and the projection of the orbital angular 


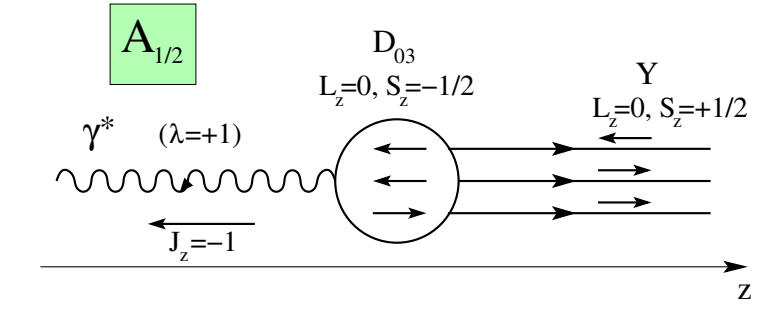

(b)

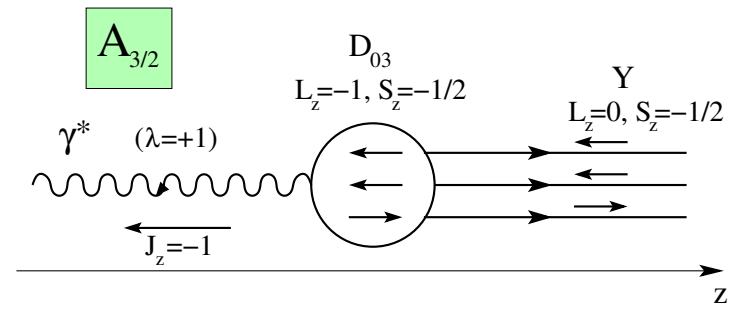

(c)

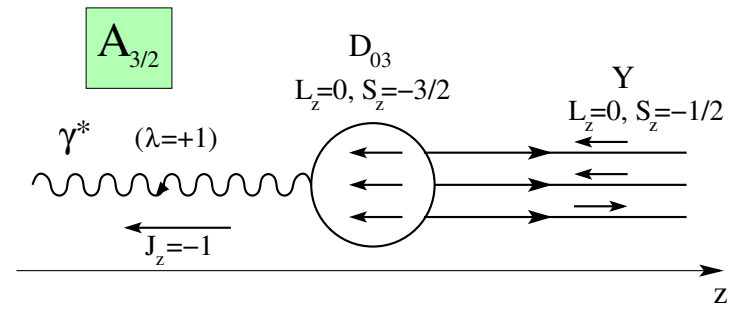

Fig. 24. A $D_{03}$-resonance in its rest frame decays electromagnetically to a ground-state hyperon $Y$. The three depicted processes refer to different contributions to the helicity amplitudes $A_{1 / 2}$ (process (a)) and $A_{3 / 2}$ (processes (b) and (c)).

momentum $\left(L_{z}\right)$ changes by 1 . As mentioned before, at high $Q^{2}$, the photon preferentially couples to the individual quarks. As a consequence, a process like the one in fig. 24(b) is suppressed relative to the one of fig. 24(a). If the BS amplitude of the resonance is dominated by $S=3 / 2 S U(6)$ states, at high $Q^{2}$ the major contribution to the $A_{3 / 2}$ comes from the process in fig. 24(c). Here, the photon is emitted by a single CQ, which accordingly flips its spin. In the situation of fig. 24(c), three CQs can emit the photon, while in fig. 24(a), only two can do that. Therefore, the $A_{3 / 2}$ can be anticipated to be larger than the $A_{1 / 2}$, resulting in negative helicity asymmetries.

A stringent test of the above-mentioned argument is provided by the helicity asymmetries of $J=3 / 2$ $\Sigma$-resonances, decaying to the $\Lambda$ and $\Sigma$ ground states. This is illustrated in fig. 25. The $P_{13}(1385)$, a member of the baryon decuplet, possesses a symmetric spin wave function. In ref. [31] it was pointed out that the $P_{13}(2080)$-resonance has an almost purely symmetric spin wave function. Both resonances display negative helicity asymmetries, even at relatively low values of $Q^{2}$, for all isospin channels. Furthermore, since only two CQs contribute to the process in fig. 24(a) and three CQs contribute to the process of fig. 24(c), one may expect a helicity asymmetry of $\frac{2^{2}-3^{2}}{2^{2}+3^{2}} \approx-0.4$. This is clearly in agreement with the left panels of fig. 25 . The computed helicity
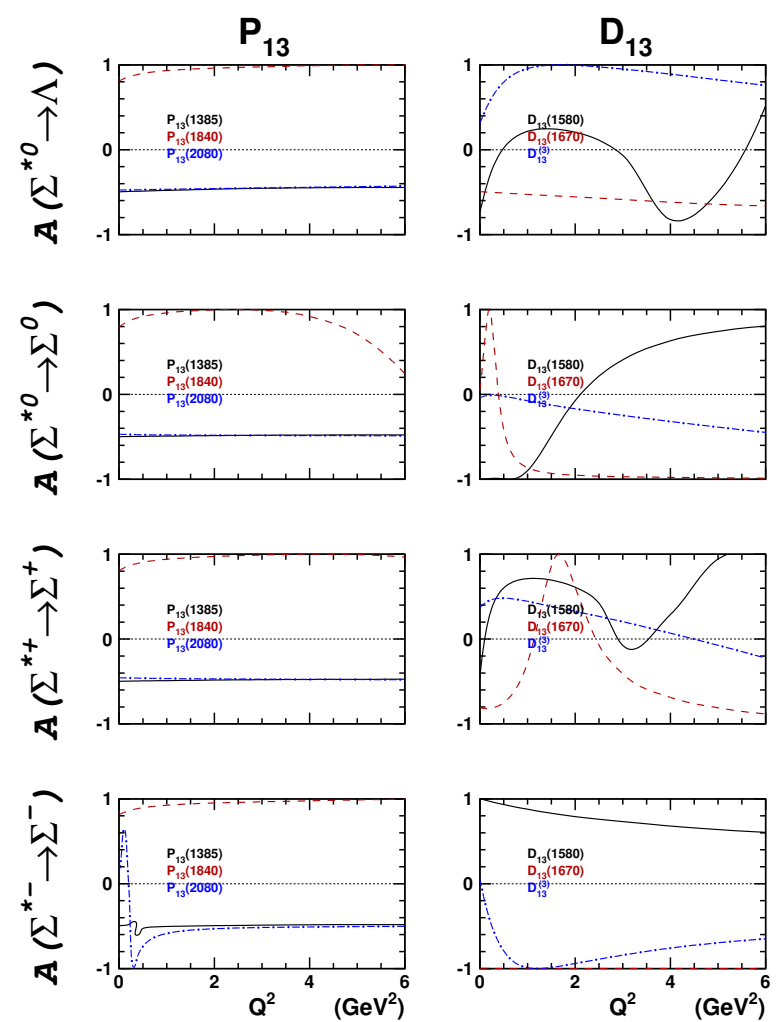

Fig. 25. The helicity asymmetry as defined in eq. (28) for the lowest-lying spin $J=3 / 2 \Sigma$-resonances decaying to the $\Lambda$ (upper row), the $\Sigma^{0}$ (second row), the $\Sigma^{+}$(third row) and the $\Sigma^{-}$(lower row) ground states.

asymmetry of the $P_{13}(1840)$ is in accordance with a purely mixed-symmetric spin wave function reported in ref. [31].

For the $D_{13}$-resonances, the situation is more complicated. The spin wave function of the $D_{13}(1580)$ is dominantly of mixed symmetry $(S=1 / 2)$, resulting in a helicity asymmetry which goes to +1 at high $Q^{2}$. The $D_{13}(1670)$ has a spin wave function which is a mixture of $S=1 / 2$ and mostly $S=3 / 2$ components, and thus displays negative helicity asymmetries. Finally, the $D_{13}^{(3)}$-resonance has a spin wave function which is a mixture of $S=3 / 2$ and mostly $S=1 / 2$ components. Its helicity asymmetries seem to depend on the isospin and charge of the octet hyperon which it decays to.

\section{Conclusions}

The Bonn CQ model has been applied to the computation of helicity amplitudes of strange-baryon resonances. The seven parameters entering the Bonn model were fitted previously to the masses of the best-known baryons $[27,31]$. Therefore, the present results for the helicity amplitudes and EM decay widths can be regarded as predictions. We have calculated the electromagnetic decays $\Lambda^{*} \rightarrow \Lambda(1116)$, $\Lambda^{*} \rightarrow \Sigma(1193), \Sigma^{*} \rightarrow \Lambda(1116)$, and $\Sigma^{*} \rightarrow \Sigma(1193)$ for the lowest-lying $\Lambda^{*}$ s and $\Sigma^{*}$ 's. 
The presented results show interesting features. The first-excited state of a certain spin and parity (and sometimes also higher-excited states) couples considerably stronger to a photon with finite virtuality $Q^{2}$ than to a real photon. Therefore, these resonances can be better studied with virtual photons. Further, the lowest-lying $\Lambda^{*}$ seems to decay preferably to the $\Lambda(1116)$, while the second- and third-excited $\Lambda^{*}$ decays preferentially to the $\Sigma^{0}(1193)$.

According to the computed helicity amplitudes, the spin $J=5 / 2 F_{05}(1820)$ and $D_{05}(1830) \Lambda$-resonances have a reasonable EM coupling to the $\Lambda(1116)$. A second resonance with $J^{\pi}=5 / 2^{-}$, the $D_{05}^{(2)}$ with a computed mass of about $2100 \mathrm{MeV}$, remains unobserved experimentally, but has larger helicity amplitudes than the first $D_{05^{-}}$ resonance. On the basis of these observations, neglecting $J=5 / 2 \Lambda^{*}$-resonances in the $u$-channel background contribution of the $p\left(\gamma^{*)}, K\right) \Lambda$ process should be done with care.

For the electromagnetic decay of a $\Sigma^{*}$-resonance to the $\Sigma$ ground state, three situations, one for each member of the $\Sigma^{*}$ isospin triplet, need to be considered. The results show that the charged states of some $\Sigma^{*}$-resonances (e.g. the $P_{11}(1660)$, the $S_{11}(1620)$ and the $D_{13}(1580)$ ) have larger helicity amplitudes than the neutral state. Therefore, these $\Sigma^{*}$-resonances are expected to affect the $p\left(e, e^{\prime} K^{0}\right) \Sigma^{+}$process more than the $p\left(e, e^{\prime} K^{+}\right) \Sigma^{0}$ process.

Our investigations lend additional support for the peculiar structure of the $S_{01}(1405)$, already pointed out in refs. $[19,32,45]$. The predicted EM decay width is much larger than what is experimentally measured, both for decay to the $\Lambda(1116)$ and to the $\Sigma(1193)$. In this respect, we would like to note that the lowest-lying $\Sigma^{*}$ with negative parity, the $S_{11}(1620)$, also has large EM decay widths to the $\Lambda(1116)$ and $\Sigma(1193)$. In contrast to the $S_{01}(1405)$, the mass of the $S_{11}(1620)$ is well reproduced by the Bonn CQ model. Furthermore, our predictions for the EM decay widths of the $D_{03}(1520)$-resonance seem to be in good qualitative agreement with the PDG values [24] and the new CLAS measurement [39]. A description of this resonance in terms of three relativistic constituent quarks seems appropriate. Our model results for the EM decay widths of the $P_{13}(1385)$ decuplet resonance are systematically larger than previous investigations have suggested. The experimental value of the $P_{13}(1385) \rightarrow P_{01}(1116)$ process [39] is in between the previous predictions and ours. More work on the decuplet $\Sigma^{*}$ hyperon seems necessary.

We find larger-than-average decay widths for the process $S_{01}(1670) \rightarrow \Sigma(1193)+\gamma$. This explains the fact that the $\bar{K} p \rightarrow \gamma \Sigma$ cross-section is roughly a factor of four larger than the one for the $\bar{K} \quad p \rightarrow \gamma \Lambda$ reaction for kaon momenta of about $750 \mathrm{MeV} / c$ (invariant mass of about $1678 \mathrm{MeV})$ [37]. Also the $D_{13}(1670)$ can give a sizeable contribution to this process.

Finally, the behaviour of the helicity asymmetries for $J \geq 3 / 2$ resonances lends support for an overall picture in which at high $Q^{2}$, the photon couples to an individual constituent quark, rather than to the baryon (resonance) as a whole.
This work is supported by the Research Council of Ghent University. B.M. and H.-R.P. acknowledge the support of the European Community-Research Infrastructure activity under the FP6 "Structuring the European Research Area" program (Hadron Physics, contract No. RII3-CT-2004-506078) and the support within the DFG SFB/TR16 "Subnuclear Structure of Matter - Elektromagnetische Anregung subnuklearer Systeme".

\section{Appendix A. Effective interactions and interaction kernels}

In this appendix, a brief description of the quark-quark interactions used in the kernel of the Bethe-Salpeter equation (2) is given. As shown in sect. 2.2, the Bethe-Salpeter equation can be reduced to a Salpeter equation if the interactions are assumed to be instantaneous. In the model, two types of interactions appear. The three-particle irreducible confinement potential will be discussed in sect. A.1. The instanton-induced two-particle irreducible residual interaction is the subject of sect. A.2.

\section{Appendix A.1. Confinement potential}

In the Bonn model, the confinement interaction is a stringlike potential which rises linearly with the interquark distances. This results in almost linear Regge trajectories for both mesons and baryons in the Bonn model.

The confinement potential is the only three-particle irreducible interaction that enters the model. It is given by

$$
\begin{aligned}
& V^{(3)}\left(x_{1}, x_{2}, x_{3} ; x_{1}^{\prime}, x_{2}^{\prime}, x_{3}^{\prime}\right)=V_{\mathrm{conf}}^{(3)}\left(\mathbf{x}_{1}, \mathbf{x}_{2}, \mathbf{x}_{3}\right) \\
& \times \delta^{(1)}\left(x_{1}^{0}-x_{2}^{0}\right) \delta^{(1)}\left(x_{2}^{0}-x_{3}^{0}\right) \delta^{(4)}\left(x_{1}-x_{1}^{\prime}\right) \\
& \times \delta^{(4)}\left(x_{2}-x_{2}^{\prime}\right) \delta^{(4)}\left(x_{3}-x_{3}^{\prime}\right) .
\end{aligned}
$$

Here, the one-dimensional $\delta$-functions of the time components implement the assumption that the interaction is instantaneous. The actual confinement potential $V_{\text {conf }}^{(3)}\left(\mathbf{x}_{1}, \mathbf{x}_{2}, \mathbf{x}_{3}\right)$ is a function of the relative quark coordinates, but also comprises Dirac structures which act on the quark spinors. It can be written as [27]

$$
V_{\text {conf }}^{(3)}=a \mathcal{W}_{\text {off }}+b r_{3 q}\left(\mathbf{x}_{1}, \mathbf{x}_{2}, \mathbf{x}_{3}\right) \mathcal{W}_{\text {str }},
$$

where $a$ and $b$ are the confinement parameters, $r_{3 q}$ is a measure for the interquark distance, and $\mathcal{W}_{\text {off }}$ and $\mathcal{W}_{\text {str }}$ are the Dirac structures operating on the constituent-quark spinors. The parameters $a$ and $b$ are the sole parameters associated with the confinement potential. These parameters and the $m_{u} \equiv m_{d} \equiv m_{n}$ nonstrange constituentquark mass are determined by optimizing the model results for the $\Delta$ spectrum to the experimentally best-known resonance masses. The optimized $a, b$ and $m_{n}$ are contained in table 8.

The interquark distance $r_{3 q}$ for three constituent quarks can be defined in different manners. We use the 


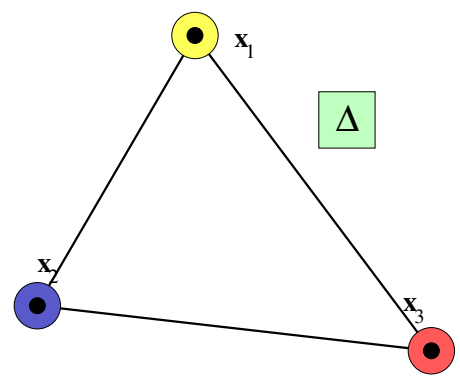

(a)

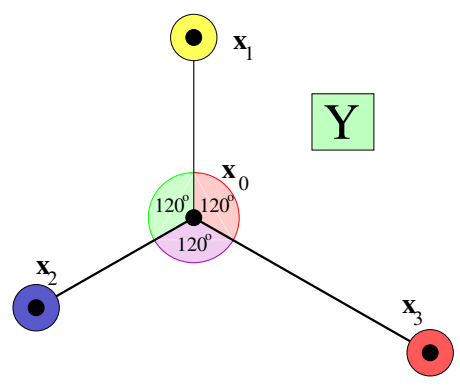

(b)

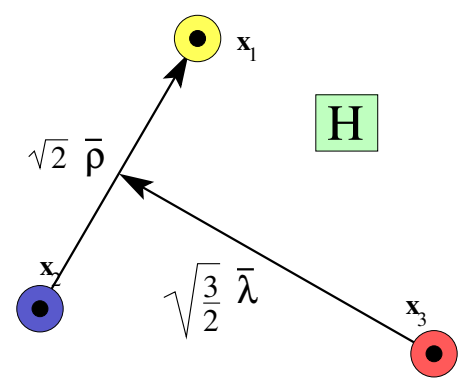

(c)

Fig. 26. The $\Delta$-, the $Y$ - and the $H$-configuration for the interquark distance $r_{3 q}$ are shown in (a), (b) and (c), respectively. In (a) and (b), the length of the connecting lines is the interquark distance. In (c), $r_{3 q}$ is given by eq. (A.5).

Table 8. The seven parameters of the Bonn model are the constituent-quark masses, the confinement offset and slope, the 't Hooft interaction range, and the 't Hooft nonstrangenonstrange and nonstrange-strange interaction strength.

\begin{tabular}{|c|c|c|c|}
\hline Parameter & Symbol & Value & Unit \\
\hline Nonstrange CQ mass & $m_{n}$ & 330 & $\mathrm{MeV}$ \\
\hline Strange CQ mass & $m_{s}$ & 670 & $\mathrm{MeV}$ \\
\hline Confinement offset & $a$ & -744 & $\mathrm{MeV}$ \\
\hline Confinement slope & $b$ & 470 & $\mathrm{MeV} \mathrm{fm}^{-1}$ \\
\hline 't Hooft $n n$ strength & $g_{n n}$ & 136 & $\mathrm{MeV} \mathrm{fm}{ }^{3}$ \\
\hline 't Hooft $n s$ strength & $g_{n s}$ & 94 & $\mathrm{MeV} \mathrm{fm}{ }^{3}$ \\
\hline 't Hooft range & $\Lambda$ & 0.40 & $\mathrm{fm}$ \\
\hline
\end{tabular}

sum of the three distances between the quarks, which is commonly referred to as a $\Delta$-configuration:

$$
r_{3 q}\left(\mathbf{x}_{1}, \mathbf{x}_{2}, \mathbf{x}_{3}\right)=\sum_{i<j}\left|\mathbf{x}_{i}-\mathbf{x}_{j}\right|
$$

In the literature, one finds alternative definitions, such as the $Y$ - and $H$-configuration as depicted in fig. 26. The $Y$-configuration uses the minimal length to connect three points:

$$
r_{3 q}\left(\mathbf{x}_{1}, \mathbf{x}_{2}, \mathbf{x}_{3}\right)=\min _{\mathbf{x}_{0}} \sum_{i<j}\left|\mathbf{x}_{i}-\mathbf{x}_{0}\right|,
$$

whereas the $H$-version puts forward the hyperradius as a measure of the interquark distance:

$$
r_{3 q}\left(\mathbf{x}_{1}, \mathbf{x}_{2}, \mathbf{x}_{3}\right)=\sqrt{|\boldsymbol{\rho}|^{2}+|\boldsymbol{\lambda}|^{2}}
$$

where $\boldsymbol{\rho}=\frac{1}{\sqrt{2}}\left(\mathbf{x}_{1}-\mathbf{x}_{2}\right)$ and $\boldsymbol{\lambda}=\frac{1}{\sqrt{6}}\left(\mathbf{x}_{1}+\mathbf{x}_{2}-2 \mathbf{x}_{3}\right)$. It turns out, however, that the slope parameter $b$ of the confinement potential can be scaled such that the results for the three variants are of equal quality. Lattice calculations seem to favor a configuration which is a mixture of the $\Delta$ and $Y$-variant [46]. Numerically, the $\Delta$-configuration is easier to handle in CQ model calculations and is the one adopted here.

The Dirac structures $\mathcal{W}_{\text {off }}$ and $\mathcal{W}_{\text {str }}$ have a large impact on the computed baryon spectrum. The specific choice for these structures is constrained by the observation that the spin-orbit effects in the baryon spectrum are moderate (note, e.g., the small mass difference between the $S_{11}(1535)$ and $D_{13}(1520)$ nucleon resonances). Furthermore, $\mathcal{W}_{\text {off }}$ and $\mathcal{W}_{\text {str }}$ can have a different structure. The best choice is $[8]$

$$
\begin{aligned}
& \mathcal{W}_{\text {off }}=\frac{3}{4}\left[\mathbb{I} \otimes \mathbb{I} \otimes \mathbb{I}+\gamma^{0} \otimes \gamma^{0} \otimes \mathbb{I}\right. \\
&+ \text { cycl. perm. }], \\
& \mathcal{W}_{\text {str }}=\frac{1}{2}[-\mathbb{I} \otimes \mathbb{I} \otimes \mathbb{I}+ \gamma^{0} \otimes \gamma^{0} \otimes \mathbb{I} \\
&+ \text { cycl. perm. }] .
\end{aligned}
$$

With this specific choice for the Dirac structures, the $V_{\text {conf }}^{(3)}$ of eq. (A.2) reduces to a spin-independent linear confinement potential in the nonrelativistic limit [8].

\section{Appendix A.2. 't Hooft Instanton-Induced Interaction}

In the Bonn model, the hyperfine splittings in the baryon spectrum are induced by a two-particle irreducible interaction based on the effects of instantons on the propagation of light quarks. Instantons are classical, nonperturbative solutions of the QCD Yang-Mills equations in Euclidean spacetime. They are localized in space and imaginary time and describe tunneling events. Instantons (anti-instantons) absorb right-handed (left-handed) lightflavoured quarks, and emit left-handed (right-handed) ones. As such they mediate a force between light quarks. Furthermore, instantons change the axial charge of the QCD vacuum in the presence of an external fermion source. Therefore, they provide an explanation for the nonconservation of axial charge. The crucial properties of instantons were discovered by 't Hooft [47]. Therefore, the resulting interaction between light quarks is sometimes referred to as the 't Hooft interaction.

The two-body part of the 't Hooft Instanton-Induced Interaction, $V_{\text {III }}^{(2)}$, induces a flavour-, spin- and colour-dependent force between two light quarks. In particular it 
acts between flavour antisymmetric quark pairs according to

$$
\begin{aligned}
& V_{\mathrm{III}}^{(2)}\left(x_{1}, x_{2} ; x_{1}^{\prime}, x_{2}^{\prime}\right)=V_{\text {t Hooft }}^{(2)}\left(\mathbf{x}_{1}-\mathbf{x}_{2}\right) \\
& \quad \times \delta^{(1)}\left(x_{1}^{0}-x_{2}^{0}\right) \delta^{(4)}\left(x_{1}-x_{1}^{\prime}\right) \delta^{(4)}\left(x_{2}-x_{2}^{\prime}\right) .
\end{aligned}
$$

The 't Hooft two-body potential, $V_{\text {'t Hooft }}^{(2)}$, is a function of the distance between the two constituent quarks $\left(\mathbf{x}_{1}-\mathbf{x}_{2}\right)$, and comprises the appropriate Dirac structure and projectors in Dirac- $(\mathcal{D})$, flavour- $(\mathcal{F})$ and colourspace $(\mathcal{C})$ :

$$
\begin{aligned}
& V_{\text {'t Hooft }}^{(2)}\left(\mathbf{x}_{1}-\mathbf{x}_{2}\right)=-4 v_{\text {reg }}\left(\mathbf{x}_{1}-\mathbf{x}_{2}\right) \\
& \times \mathcal{P}_{S_{12}=0}^{\mathcal{D}} \otimes\left(g_{n n} \mathcal{P}_{\mathcal{A}}^{\mathcal{F}}(n n)+g_{n s} \mathcal{P}_{\mathcal{A}}^{\mathcal{F}}(n s)\right) \otimes \mathcal{P}_{\overline{3}}^{\mathcal{C}} \\
& \times\left(\mathbb{I} \otimes \mathbb{I}+\gamma^{5} \otimes \gamma^{5}\right) .
\end{aligned}
$$

Here, $v_{\text {reg }}\left(\mathbf{x}_{1}-\mathbf{x}_{2}\right)$ is a regulating function, describing the three-dimensional extension of the interaction:

$$
v_{\text {reg }}(\mathbf{x})=\frac{1}{\Lambda^{3} \pi^{\frac{3}{2}}} e^{-\frac{|\mathbf{x}|^{2}}{\Lambda^{2}}} .
$$

The range of the interaction, $\Lambda$, is a free parameter in the Bonn model. It is extracted from a fit of the model results to the best-known nucleon masses, and its value is listed in table 8 . The magnitude of $\Lambda$ corresponds roughly to the average size of the instanton $[27,48]$. The two interaction strengths $g_{n n}$ and $g_{n s}$, associated with the antisymmetric nonstrange-nonstrange and the strange-nonstrange flavour projectors, are also fitting parameters. The $g_{n n}$ coupling strength is fitted to the nucleon spectrum, and reproduces the hyperfine splitting between the nucleon and $\Delta(1232)$-resonance. In contrast to the nucleon, the $\Delta(1232)$ has a symmetric spin wave function. Therefore, the $V_{\mathrm{III}}^{(2)}$ of eq. (A.7) affects only the nucleon, lowering its mass compared to the $\Delta$-resonance. The strangenonstrange coupling $\left(g_{n s}\right)$ and the strange constituentquark mass $\left(m_{s}\right)$ parameters are determined in order to reproduce the masses of the experimentally best-known hyperons. As a matter of fact, the $g_{n s}$ coupling is responsible for the $\Sigma_{\text {(dec.) }}^{*}-\Sigma_{\text {(oct.) }}$ and $\Xi_{(\text {dec. })}^{*}-\Xi_{\text {(oct.) }}$ mass splittings. Values of $g_{n n}, g_{n s}$ and $m_{s}$ are listed in table 8.

\section{References}

1. S. Janssen, J. Ryckebusch, W. Van Nespen, D. Debruyne, T. Van Cauteren, Eur. Phys. J. A 11, 105 (2001); S. Janssen, J. Ryckebusch, D. Debruyne, T. Van Cauteren, Phys. Rev. C 65, 015201 (2002).

2. S. Janssen, J. Ryckebusch, D. Debruyne, T. Van Cauteren, Phys. Rev. C 66, 035202 (2002).

3. S. Janssen, J. Ryckebusch, T. Van Cauteren, Phys. Rev. C 67, R052201 (2003).

4. R.M. Mohring et al., Phys. Rev. C 67, 055205 (2003); The CLAS Collaboration (J.W.C. McNabb et al.), Phys. Rev. C 69 R042201 (2004).

5. The LEPS Collaboration (R.G.T. Zegers et al.), Phys. Rev. Lett. 91, 092001 (2003).

6. K.-H. Glander et al., Eur. Phys. J. A 19, 251 (2004); R. Lawall et al., Eur. Phys. J. A 24275 (2005).
7. T. Van Cauteren, D. Merten, J. Ryckebusch, T. Corthals, S. Janssen, B. Metsch, H.-R. Petry, Eur. Phys. J. A 20, 283 (2004).

8. U. Löring, A Covariant Quark Model of Baryons with Instanton-induced Forces, $\mathrm{PhD}$ Thesis, Rheinische Friedrich-Wilhelms-Universität Bonn, Germany (2001).

9. U. Löring, K. Kretzschmar, B. Metsch, H.-R. Petry, Eur. Phys. J. A 10, 309 (2001).

10. D. Merten, U. Löring, K. Kretzschmar, B. Metsch, H.-R. Petry, Eur. Phys. J. A 14, 477 (2002).

11. M. Koll, R. Ricken, D. Merten, B.C. Metsch, H.-R. Petry, Eur. Phys. J. A 9, 73 (2000).

12. J.W. Darewych, M. Horbatsch, R. Koniuk, Phys. Rev. D 28, 1125 (1983).

13. E. Kaxiras, E.J. Moniz, M. Soyeur, Phys. Rev. D 32, 695 (1985).

14. M. Warns, W. Pfeil, H. Rollnik, Phys. Lett. B 258, 431 (1991).

15. Y. Umino, F. Myhrer, Phys. Rev. D 39, 3391 (1989); Nucl. Phys. A 529, 713 (1991); 554, 593 (1993).

16. D.B. Leinweber, T. Draper, R.M. Woloshyn, Phys. Rev. D 48, 2230 (1993).

17. M.N. Butler, M.J. Savage, R.P. Springer, Phys. Lett. B 304, 353 (1993).

18. C.L. Schat, C. Gobbi, N.N. Scoccola, Phys. Lett. B 356, 1 (1995).

19. C.L. Schat, N.N. Scoccola, C. Gobbi, Nucl. Phys. A 585, 627 (1995).

20. A. Abada, H. Weigel, H. Reinhardt, Phys. Lett. B 366, 26 (1996).

21. G. Wagner, A.J. Buchmann, A. Faessler, Phys. Rev. C 58, 1745 (1998).

22. G. Wagner, A.J. Buchmann, A. Faessler, J. Phys. G 26, 267 (2000).

23. R.F. Lebed, D.R. Martin, Phys. Rev. D 70, 057901 (2004).

24. S. Eidelman et al., Phys. Lett. B 592, 1 (2004).

25. A. Le Yaouanc, Ll. Oliver, O. Pène, J.-C. Raynal, Hadron Transitions in the Quark Model (Gordon and Breach Science Publishers, New York, London, Paris, Montreux, Tokyo, Melbourne, 1988).

26. E.E. Salpeter, H.A. Bethe, Phys. Rev. 84, 1232 (1951).

27. U. Löring, B. Metsch, H.-R. Petry, Eur. Phys. J. A 10, 395 (2001).

28. D. Merten, Hadron Form Factors and Decays, PhD Thesis, Rheinische Friedrich-Wilhelms-Universität Bonn, Germany (2002).

29. S. Capstick, Phys. Rev. D 47, 2864 (1992); F.E. Close, Z. Li, Phys. Rev. D 42, 2194 (1990).

30. R.C.E. Devenish, T.S. Eisenschitz, J.G. Korner, Phys. Rev. D 14, 3063 (1976).

31. U. Löring, B. Metsch, H.-R. Petry, Eur. Phys. J. A 10, 447 (2001).

32. D. Jido, J.A. Oller, E. Oset, A. Ramos, U.G. Meissner, Nucl. Phys. A 725, 181 (2003); 755, 669 (2005); C. GarcíaRecio, J. Nieves, E. Ruiz Arriola, M.J. Vicente Vacas, Phys. Rev. D 67, 076009 (2003).

33. C. García-Recio, M.F.M. Lutz, J. Nieves, Phys. Lett. B 582, 49 (2004); E.E. Kolomeitsev, M.F.M. Lutz, Phys. Lett. B 585, 243 (2004).

34. S. Capstick, B.D. Keister, Phys. Rev. D 51, 3598 (1995).

35. M. Warns, W. Pfeil, H. Rollnik, Phys. Rev. D 42, 2215 (1990). 
36. S. Janssen, Strangeness Production on the Nucleon, $\mathrm{PhD}$ Thesis, Ghent University, Belgium (2002).

37. S. Prakhov, Progress on study of $K^{\mp}$ proton reactions, UCLA, USA (2001), unpublished, http://bmkn8. physics.ucla.edu/Crystalball / Docs / documentation. html.

38. D.M. Manley et al., Phys. Rev. Lett. 88, 012002 (2002).

39. The CLAS Collaboration (S. Taylor et al.), Phys. Rev. C 71, 054609 (2005).

40. N. Phaisangittisakul, First Measurement of the Radiative Process $K^{-} p \rightarrow \Lambda \gamma$ at Beam Momenta 520-750 MeV/c Using the Crystal Ball Detector, PhD Thesis, University of California, Los Angeles, USA (2001).

41. N. Isgur, G. Karl, R. Koniuk, Phys. Rev. D 25, 2394 (1982).

42. A.J. Buchmann, E.M. Henley, Phys. Rev. D 65, 073017 (2002).
43. M. Fiolhais, B. Golli, S. Širca, Phys. Lett. B 373, 229 (1996); S.S. Kamalov, Shin Nan Yang, Phys. Rev. Lett. 83, 4494 (1999); V. Pascalutsa, M. Vanderhaeghen, arXiv:hep$\mathrm{ph} / 0508060$.

44. W. Greiner, B. Müller, Quantum Mechanics - Symmetries, 2nd edition (Springer, New York, Berlin, Heidelberg, 1994).

45. T. Hyodo, A. Hosaka, M.J. Vicente Vacas, E. Oset, invited talk at the YITP Workshop on Multi-quark Hadrons; Four, Five and More?, Kyoto, Japan (2004), arXiv:nuclth/0404031.

46. G.S. Bali, Phys. Rep. 343, 1 (2001); Ph. de Forcrand, O. Jahn, Proceedings of the 10th International Conference on the Structure of Baryons (BARYONS 2004), Palaiseau, France, Nucl. Phys. A 755, 475 (2005).

47. G. 't Hooft, Phys. Rev. D 14, 3432 (1976).

48. D. Diakonov, Prog. Part. Nucl. Phys. 51, 173 (2003); E. Shuryak, Nucl. Phys. B 203, 93 (1982). 